\title{
Stability Analysis of a Breathing Cracked Rotor with Imposed Mass Eccentricity
}

\author{
Huichun Peng, ${ }^{1,2}$ Qing He, ${ }^{2}$ and Yaxin Zhen ${ }^{1}$ \\ ${ }^{1}$ Department of Mathematics and Physics, North China Electric Power University, Beijing 102206, China \\ ${ }^{2}$ School of Energy Power and Mechanical Engineering, China Electric Power University, Beijing 102206, China \\ Correspondence should be addressed to Huichun Peng; phuich@ncepu.edu.cn
}

Received 14 April 2015; Revised 8 June 2015; Accepted 15 June 2015

Academic Editor: Sundararajan Natarajan

Copyright (c) 2015 Huichun Peng et al. This is an open access article distributed under the Creative Commons Attribution License, which permits unrestricted use, distribution, and reproduction in any medium, provided the original work is properly cited.

\begin{abstract}
The investigation of the effects of mass eccentricity on stability of a gravity dominated cracked Jeffcott rotor would generally provide practical applicability to crack detection and instability control of the heavy loading turbo-machinery system. Based upon the numerical Floquet method, the stability and bifurcations of the periodic time-dependent rotor with a transverse breathing crack are studied with respect to the varied mass eccentricity at different rotation speed, and the stability diagrams in the parameter plane are obtained which the previous studies have not covered. The numerical response of the cracked rotor system is also analyzed by the frequency spectrum to present the vibration characters while the rotation speed approaches the critical ratio. The detailed numerical eigenvalues of the transition matrix are applied to analyze the types of the bifurcations of the cracked rotor system. Three types of bifurcations are found and responses of the cracked rotor system at these bifurcations are presented for the visualized comparisons.
\end{abstract}

\section{Introduction}

Fatigue cracks of rotating machinery shaft might lead to catastrophic accidents. Dynamic characteristics of cracked rotor have been studied in the last three decades $[1,2]$. Many factors can affect the stability of the cracked rotor system, such as crack depth ratio, crack position, the system damping ratio, mass eccentricity, and unbalance orientation angle. Although there are many researches of the influential parameters such as the crack depth ratio and damping ratio [3-8], as for heavy loading turbo-machinery system, the breathing cracked rotor coupled with mass eccentricity, which may excite abundance unstable behavior and bifurcations during the horizontal rotating motion, is in need of consideration.

As the motion equations of the cracked rotor systems are nonlinear and nonautonomous differential equations due to the open-close of the crack during the rotation of the rotor, the response is analyzed by means of the qualitative theory of nonlinear dynamics [9]. In the survey of Genesio [10], the existing methods on estimation of the region of asymptotic stability for continuous, autonomous, and nonlinear systems are mainly the perturbation methods to numerically approach to stability border lines. But owing to theoretical idealized setting, the approximation appears to be quite hard to be applied to more complicated rotor models with multivaried parameters.

Based on the stability analyses of higher linear differential equations with periodic coefficients [11], Meng and Gasch [3] and Untaroiu et al. [4] explored its applications to cracked rotor system. The transition matrix approach based on Floquet theorem, instead of perturbation methods, was employed to make the analysis of stability of a cracked rotor system. By means of evaluating the eigenvalues of the Floquet transition matrix, Guo et al. [5] investigated the effect of damping on the instability regions of a cracked rotor system in the complex plane, and Chu et al. [6-8] examined the effects of crack depth and position on the instability of multidisk cracked rotors and slant cracked rotors. The works show that the stability degree (determined by the eigenvalues of the Floquet transition matrix) can act as an efficient index to analyze the stability and bifurcation of a cracked rotor system.

On the other hand, in the field of numerical simulations of the response of a cracked rotor, scientists tried to find 
some more efficient ways to express the breathing motion of the rotating cracked rotor. There are primarily two types of cracks which are studied in the literature. They are called both open crack and breathing crack model [12-16]. The open crack model, which is considered to be more suitable for the small mass rotor (less than $10 \mathrm{~kg}$ ) with a relatively high rotation speed, is proposed as the stiffness asymmetry rotors approached by Chu et al. $[7,17]$ and Genesio et al. [10]. As for the crack on the large quantity (larger than $20 \mathrm{~kg}$ ) rotors, which are normally operating on a relatively low speed, usually breath from closed state in the compression zone to open state in tension zone is the case for breathing crack model $[15,16]$.

Bachschmid et al. [12-15] proposed the breathing cracked model as the coefficients of the Fourier series based on the finite element models to simulate the dynamic response of the actual experimental rotors. But the complicate calculation limits its application in the Jeffcott model. Mayes and Davies [16] presented a classical form for the breathing crack function to simulate the actual breathing behavior, which is assumed to be a more feasible approximation process for the cracked rotor systems.

Although the breathing mechanism in rotating shafts can be accurately reproduced by $3 \mathrm{D}$ nonlinear finite element models, Bachschmid et al. $[13,14]$ showed that a simple approximate model can also accurately simulate the breathing behavior of the rotating cracked rotor. Han [18] and Patel and Darpe [19] showed that the method based on fracture mechanics, using stress intensity factors and the energy release rate to calculate the stiffness, combined with a breathing function, can increase the efficiency of the dynamic analysis. Bifurcation diagram, orbit plot, and Poincare map were employed to investigate the numerical simulation of transient response of the cracked rotor system to find the nonlinear dynamic characteristic of the cracked rotor [2024] based on the two basic model of open-close cracked rotor, both switching and breathing models. These studies exhibited the splendid visualized comparisons and provide the nonlinear signals directly for monitoring and diagnosing fault of rotating machines. The nonlinear signals of the harmonically varying stiffness breathing models were showed to be in high compliance with the actual experimental rotors.

In view of the accurate actual experimental crack model, the previous researches of stability analyses of multidegreeof-freedom rotor system are mainly limited to investigate the influence of the crack depth ratio and the system damping ratio on the stability of cracked rotor system [3-8, 17, 25]. In fact, there are many other factors influencing the stability degree and stable region of the vibration dynamic of a rotating cracked rotor. Mass eccentricity is another important parameter that has significant influence on the stability of gravity dominated cracked rotor dynamic system. In practical experience, the mass eccentricity can be detected and modified by attaching an additional massive on the rotating machinery shaft. The research on the influence of varied mass eccentricity on the stability and bifurcations at the critical value of stability degree of cracked rotor is necessary to improve the understanding of the nonlinear dynamic characters of the cracked rotor system and consequently may help in the identification of cracks in rotating machinery. The study is presented as follows.

Firstly, we facilitate the evaluation of the transition matrix by calculating the stability degree of periodic vibration based on transient response. Secondly, in order to generate the transient response of the cracked rotor system in high compliance with the actual experimental rotors, the crack model is simulated by using stress intensity factors and the energy release rate to calculate the harmonically varying stiffness combined with a breathing function to describe the breathing motion during the rotating of the cracked rotor with weight dominance. Consequently, we apply the improved numerical transition matrix approach method to examine the dynamic response of a rotating rotor with a transverse breathing crack. We exhibit the influence of the varied mass eccentricity on the stability of the breathing cracked rotor system in different rotation speed in a stability diagram in the parameter plane. The numerical response of the cracked rotor system is also analyzed by the frequency spectrum to present the vibration characters, as the rotation speed approach the critical rotation speed, to view the differences between the dynamics of cracked and uncracked shaft which may cause the change of stabilities. Finally, the detailed numerical eigenvalues of the transition matrix are applied to analyze the types of the bifurcations in the response of the cracked rotor system. Three types of bifurcations are found and responses of the cracked rotor system at these bifurcations are presented to for the visualized comparisons.

\section{Transition Matrix Approach Method Based on the Floquet Theory}

2.1. Stability Degree of Periodic System. Given a nonlinear nonautonomous dynamic system described by the equation,

$$
\frac{d x}{d t}=F(x, t)
$$

$(x, t) \in R^{n} \times R, x$ is the state vector, and $F(x, t)$ is a periodic function of $t$ with the rotation period T. Suppose that $x^{*}(t)$ is the periodic solution of this nonlinear system with period $T$. The linear approximate disturbing equation is obtained by putting a perturbation $\delta x(t)$ to $x^{*}(t)$ where $x(t)=x^{*}(t)+$ $\delta x(t)$

$$
\delta \dot{x}=A(t) \delta x(t),
$$

where $A(t+T)=A(t)$. Suppose that $\Phi(t)$ is the basic solution matrix of (2); by the Floquet theory [10, 11], we have

$$
\Phi(t+T)=\Phi(t) B
$$

where $B$ is the Floquet matrix. Then all solutions of (2) can be expressed as

$$
\begin{aligned}
\varphi(t+T) & =B \varphi(t)=\Phi(t+T) \Phi^{-1}(t) \varphi(t) \\
& =\Phi(t) \Phi^{-1}(t) \varphi(t)
\end{aligned}
$$


Then the perturbation $\delta x(t)$ to $x^{*}(t)$ is

$$
\begin{gathered}
\delta x(t+T)=\Phi(t) B \Phi^{-1}(t) \delta x(t), \\
\delta x(t+2 T)=\Phi(t) B \Phi^{-1}(t) \delta x(t+T), \\
\vdots \\
\delta x(t+n T)=\Phi(t) B \Phi^{-1}(t) \delta x(t+(n-1) T) ;
\end{gathered}
$$

let $S=[\delta x(t+T), \ldots, \delta x(t+n T)][\delta x(t), \ldots, \delta x(t+(n-$ $1) T)]^{-1}$; from (5), we have $S=\Phi(t) B \Phi(t)^{-1}$. Matrix $S$ has same eigenvalues with the Floquet matrix $B$, which are called Floquet multipliers. By [9-11], we know the fact that the system is Lyapunov stable if the modulus of all multipliers are less than or equal to 1 (the algebraic multipliers of all the eigenvalues with modulus 1 equal to their geometric multipliers) and unstable if there is at least one of the Floquet multipliers that has modulus greater than one. as [11]

The stability degree of the periodic system (1) is defined

$$
\lambda=-\frac{1}{T} \ln \left(\max \left|\mu_{i}\right|\right),
$$

where $\mu_{i}(i=1 \cdots n)$ are Floquet multipliers, which are eigenvalues of the discrete state transition matrix $S(t)$. It is obvious that the stability degree acts as an index to point out the stability of the periodic motion. The system is stable as $\lambda>0$ and unstable as $\lambda<0$. $\lambda=0$ denote a critical state of the system. We can use a simplified numerical method to determine the characteristic multipliers in practical computation as follows.

2.2. A Numerical Method of Calculation of State Transition Matrix of Periodic Solution. To express $\delta x(t)$ by $x(t)$ will be more convenient in the measurement in engineering. Considering the periodic property of $x^{*}(t)$, we have the following equations:

$$
\begin{gathered}
x(t)=x^{*}(t)+\delta x(t), \\
x(t+T)=x^{*}(t)+\delta x(t+T), \\
\vdots \\
x(t+n T)=x^{*}(t)+\delta x(t+n T) .
\end{gathered}
$$

Obviously,

$$
\begin{aligned}
H(k) & =\delta x(t+(k+1) T)-\delta x(t+k T) \\
& =x(t+(k+1) T)-x(t+k T)
\end{aligned}
$$

for $k=0, \ldots, n$. Considering (5), we have

$$
H(k+1)=\Phi(t) B \Phi(t)^{-1} H(k) .
$$

And the eigenvalues of matrix $\Gamma(t)=[H(1), \ldots, H(n)][H(0)$, $\ldots, H(n-1)]^{-1}$ are also the Floquet multipliers. The information of $x(t)$ can be obtained by numerical integration of

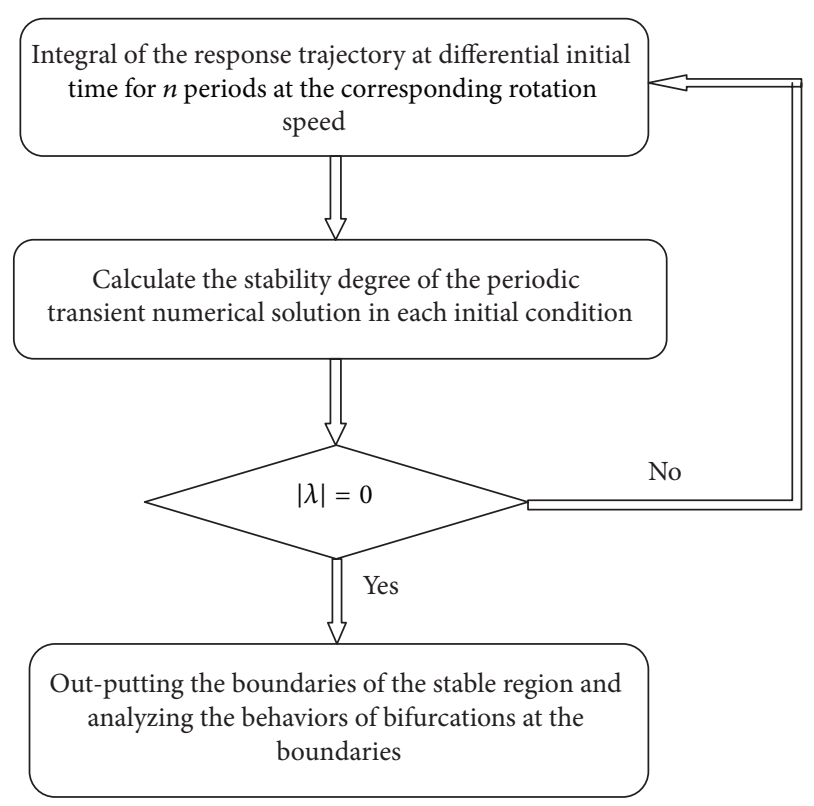

FIGURE 1: Procedures in estimation of stability of a nonlinear dynamic system.

the motion equations of the rotor system or be measured in practical engineering. So it will be a convenient method to evaluate the Floquet multipliers based on transient response. The procedures in estimation of stability of a nonlinear dynamic system based on the transient response are shown as the following strategies in Figure 1.

\section{Crack Breathing Models}

Figure 2 shows a Jeffcott rotor of which having the disc mass $m$ in the center $o$ of the shaft with length $L$ is assumed to be fixed on rigid bearing supports. The transverse crack in the middle of the span of the shaft with depth $a$ is deemed to be smooth on the edge. $o X Y Z$ are the stationary coordinates, where $o X$ is the axial direction and $o-Y Z$ is the stationary coordinates in the cross section plane of the cracked shaft. $o-$ $\xi \eta$ is the rotating coordinates in the crack cross section where $o \xi$ is supposed to be the crack direction. $\varepsilon$ is the unbalance eccentricity of the disc; $\beta$ is the unbalanced oriented angle measured from the crack direction $o \xi$; and $\Omega$ is the rotation speed.

The crack on the shaft is assumed to be breathing from completely close in the compression zone to open gradually in tension zone. Different from the rough approach to the breathing behavior of the rotating cracked rotor, switch model, on which the crack is assumed to be in either fully open state or fully close state, the breathing crack model takes the partial opening and closing state into account $[9,13,16$, $18,19]$. In the breathing crack model, the stiffness of a shaft varies periodically during the revolution with the domination of gravitational force and the unbalance deviation from crack plane.

3.1. The Stiffness Models for a Cracked Rotor. Suppose that $k_{0}$ is the stiffness of the uncracked shaft, and the reduced amount 


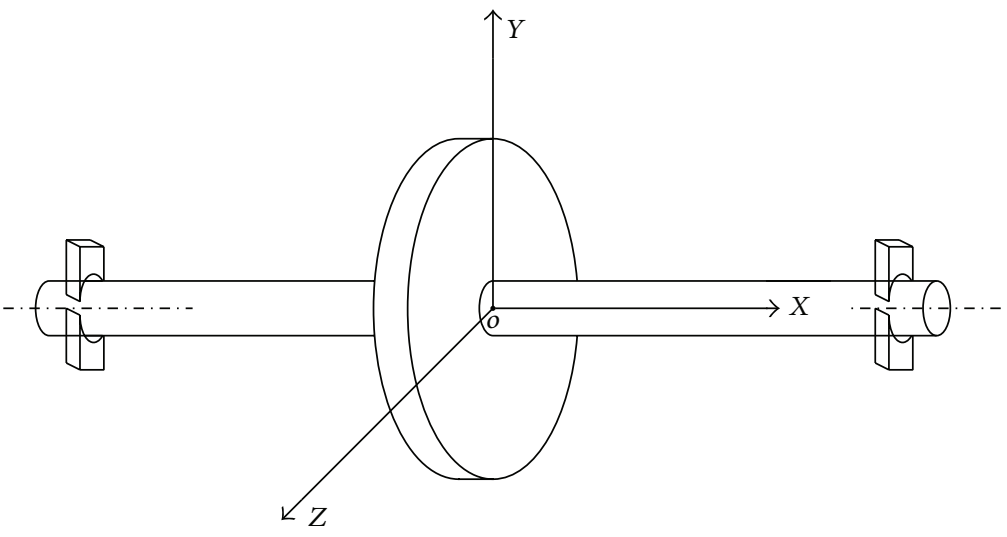

(a)

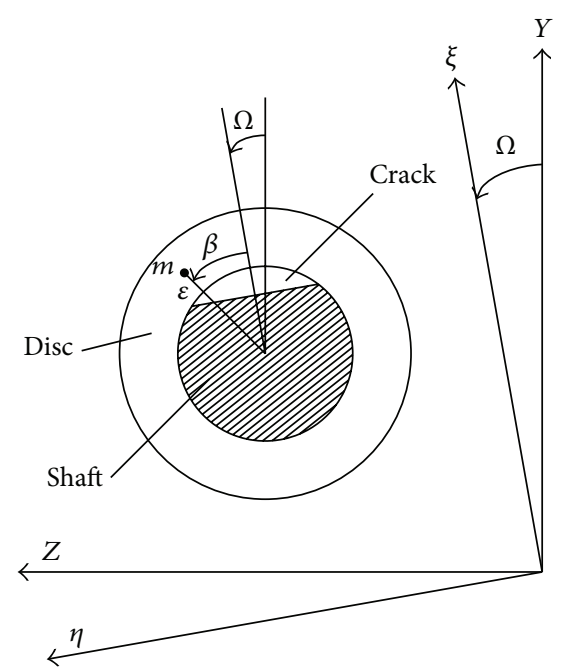

(b)

FIGURE 2: Stationary and rotational coordinates of a crack in a Jeffcott rotor.

of the stiffness in $\xi$ and $\eta$ directions is $\Delta k_{\xi}$ and $\Delta k_{\eta}$, and then the stiffness of the cracked shaft in the rotational coordinates can be expressed as follows:

$$
\left(\begin{array}{cc}
k_{\xi} & 0 \\
0 & k_{\eta}
\end{array}\right)=\left(\begin{array}{cc}
k_{0} & 0 \\
0 & k_{0}
\end{array}\right)-\Xi(\xi)\left(\begin{array}{cc}
\Delta k_{\xi} & 0 \\
0 & \Delta k_{\eta}
\end{array}\right)
$$

where $\Delta k_{\xi}=k_{0}-\widehat{k}_{\xi}$ and $\Delta k_{\eta}=k_{0}-\widehat{k}_{\eta} \cdot \widehat{k}_{\xi}$ and $\widehat{k}_{\eta}$ are the stiffness of the cracked shaft in $\xi$ and $\eta$ direction. Switch function is denoted by $\Xi(\xi)$ to describe the breathing motion of the cracked rotor.

In the actual operation, switched function jumps from 1 to 0 to express the motion of the cracked rotating rotor from the fully opened crack to fully close crack. The response in $\xi$ direction is the independent variable of the switch function, $\Xi(\xi)=\operatorname{sign}(\xi)$, which means that the crack is open when the response of rotor centre along crack direction $\xi$ is positive and closed when it is negative.

Although the switching model is the simplest expression of a breathing crack $[4,19,24,25]$, it is considered that the switching model is more adaptive for the very small cracks than a common case $[17,21,24]$. In addition, the switch function is response-dependent function resulting nonlinear motion equations which are too abrupt to be analysed as a periodic-time dependent system.

Utilizing the model methods, a harmonic model proposed by Mayes and Davies [16] expresses the smooth transition between the opening and closing of the breathing crack, $\Xi(\Omega)=(1+\cos (\Omega+\beta)) / 2$, where $\Omega=\omega(t)$, which is assumed to be the suitable model for the larger cracks, and the partial opening and closing states are also taken into account.

Cheng et al. [26] proposed a the breathing function based on the strain energy release rate method, which can be expressed as higher order harmonics of $(1+\cos (\Omega+\beta))$, and the nonlinear signals of the higher order harmonically varying stiffness breathing models is showed to be highly compliance with the actual experimental rotors $[18,19,26]$.

Therefore we use the 5th order harmonic model to simulate the breathing motion of the cracked rotor system as the following form:

$$
\begin{aligned}
\Xi(\Omega)= & \frac{1+\cos (\Omega+\beta)}{2}-4.012\left(\frac{1+\cos (\Omega+\beta)}{2}\right)^{3} \\
& +9.913\left(\frac{1+\cos (\Omega+\beta)}{2}\right)^{5}
\end{aligned}
$$

Then the stiffness matrix in the stationary coordinates can be expressed as follows:

$$
K=T^{-1}\left(\begin{array}{cc}
k_{\xi} & 0 \\
0 & k_{\eta}
\end{array}\right) T
$$

where the transformation matrix is $T=\left(\begin{array}{cc}\cos (\Omega) & \sin (\Omega) \\ -\sin (\Omega) & \cos (\Omega)\end{array}\right)$ and the periodic time-dependent form of stiffness matrix is simplified as follows:

$$
K=\left(\begin{array}{cc}
k_{0} & 0 \\
0 & k_{0}
\end{array}\right)-\frac{1}{2} \Xi(\Omega)\left(\begin{array}{cc}
2 k_{0}-\widehat{k}_{\xi}-\widehat{k}_{\eta}+\left(\widehat{k}_{\eta}-\widehat{k}_{\xi}\right) \cos (2 \Omega) & \left(\widehat{k}_{\eta}-\widehat{k}_{\xi}\right) \sin (2 \Omega) \\
\left(\widehat{k}_{\eta}-\widehat{k}_{\xi}\right) \sin (2 \Omega) & 2 k_{0}-\widehat{k}_{\xi}-\widehat{k}_{\eta}-\left(\widehat{k}_{\eta}-\widehat{k}_{\xi}\right) \cos (2 \Omega)
\end{array}\right)
$$


3.2. The Equations of Motion of a Rotating Cracked Rotor. The equations of motion of a rotating cracked rotor with the rotation speed $\Omega$ are constructed under the disk stationary coordinates as shown in the following $[9,16]$ :

$$
M \ddot{x}+C \dot{x}+K x=G+f,
$$

where $x$ is the position vector $x=\left(\begin{array}{c}Y \\ Z\end{array}\right) ; M$ is the mass matrix of rotor system $M=\left(\begin{array}{cc}m & 0 \\ 0 & m\end{array}\right)$; $C$ is the damping matrix $C=$ $\left(\begin{array}{ll}c & 0 \\ 0 & c\end{array}\right) ; K$ is the stiffness matrix showed in (13); $G=\left(\begin{array}{c}-m g \\ 0\end{array}\right)$, and $f=m \varepsilon \omega^{2}\left(\begin{array}{c}\cos (\Omega+\beta) \\ \sin (\Omega+\beta)\end{array}\right)$ where $\varepsilon$ is the unbalance eccentricity of disc.

We simplify the above equations to the dimensionless form as follows:

$$
\begin{aligned}
& \ddot{\bar{x}}+2 \zeta \dot{\bar{x}}+\left[\left(\begin{array}{cc}
(\iota)^{2} & 0 \\
0 & (\iota)^{2}
\end{array}\right)\right. \\
& \left.\quad-\Xi(\Omega)\left(\begin{array}{cc}
\delta_{1}+\delta_{2} \cos (2 \Omega) & \delta_{2} \sin (2 \Omega) \\
\delta_{2} \sin (2 \Omega) & \delta_{1}-\delta_{2} \cos (2 \Omega)
\end{array}\right)\right] \bar{x} \\
& =e\left(\begin{array}{c}
\cos (\Omega+\beta) \\
\sin (\Omega+\beta)
\end{array}\right)-\left(\begin{array}{c}
(\iota)^{2} \\
0
\end{array}\right)
\end{aligned}
$$

by using following nondimensional parameters:

$$
\bar{x}=\left(\frac{\bar{Y}}{Z}\right)=\left(\begin{array}{c}
Y / \sigma \\
Z / \sigma
\end{array}\right), \zeta=c / 2 \sqrt{k_{0} m}, \Omega=\omega t, e=\varepsilon / \sigma, \delta_{1}=
$$
$2(\iota)^{2}-\left(\delta_{\xi}\right)^{2}-\left(\delta_{\eta}\right)^{2}, \delta_{2}=\left(\delta_{\eta}\right)^{2}-\left(\delta_{\xi}\right)^{2}, \iota=\omega_{0} / \omega, \delta_{\xi}=\omega_{\xi} / \omega$, $\delta_{\eta}=\omega_{\eta} / \omega$, where $\sigma=m g / k_{0}, \omega_{0}=\sqrt{k_{0} / m}, \omega_{\xi}=\sqrt{\widehat{k}_{\xi} / m}$, $\omega_{\eta}=\sqrt{\widehat{k}_{\eta} / m}$.

\section{Dynamic Analysis of Cracked Rotors}

The dimensionless equations of motion equation (15) is solved by Runge-Kutta fourth-order numerical integration method with physical rotor parameters [17-19] for the numerical simulations shown as follows: shaft diameter, $D=$ $135 \mathrm{~mm}$; length, $L=627 \mathrm{~mm}$; disk mass, $m=34.2 \mathrm{~kg}$; damping ratio, $\zeta=0.01$.

The stiffness coefficients in the rotational frame are calculated by the flexibility values of shaft [27], as follows:

$$
\begin{aligned}
& \widehat{k}_{\xi}=\frac{C_{\eta}}{C_{\xi} C_{\eta}-\left(C_{\xi \eta}\right)^{2}}, \\
& \widehat{k}_{\eta}=\frac{C_{\xi}}{C_{\xi} C_{\eta}-\left(C_{\eta \xi}\right)^{2}},
\end{aligned}
$$

where $C_{\xi}$ and $C_{\eta}$ are the flexibility of the cracked shaft in $\xi$ and $\eta$ direction and $C_{\xi \eta}$ and $C_{\eta \xi}$ is the the coupling flexibility:

$$
\begin{aligned}
C_{\xi} & =C_{0}+\frac{32}{D^{2}} \int_{-h / D}^{h / D} \int_{0}^{a / D}\left[1-\left(\frac{2 w}{D}\right)^{2}\right]\left(\frac{2 \alpha}{D}\right) \\
\cdot & F_{1}^{2}\left(\frac{\alpha}{h}\right) d w d \alpha, \\
C_{\eta} & =C_{0}+\frac{64}{D^{2}} \int_{0}^{h / D} \int_{0}^{a / D}\left(\frac{2 w}{D}\right)^{2}\left(\frac{2 \alpha}{D}\right) \\
\cdot & F_{2}^{2}\left(\frac{\alpha}{h}\right) d w d \alpha, \\
C_{\xi \eta} & =C_{\eta \xi}=\frac{32}{D^{2}} \int_{-h / D}^{h / D} \int_{0}^{a / D} \sqrt{1-\left(\frac{2 w}{D}\right)^{2}}\left(\frac{2 w}{D}\right) \\
\cdot & \left(\frac{2 \alpha}{D}\right)\left(F_{1}\left(\frac{\alpha}{h}\right)\right)\left(F_{2}\left(\frac{\alpha}{h}\right)\right) d w d \alpha,
\end{aligned}
$$

where $h=\sqrt{D^{2}-4 w^{2}}$. And $C_{0}=L^{3} / 48 E I$ is flexibility of the uncracked shaft, and $F_{1}$ and $F_{2}$ are the geometry correction factor (Figure 3):

$$
\begin{aligned}
& F_{1}\left(\frac{\alpha}{h}\right)=\frac{\sqrt{(2 h / \pi \alpha) \tan (\pi \alpha / 2 h)}\left[0.752+2.02(\alpha / h)+0.37(1-\sin (\pi \alpha / 2 h))^{3}\right]}{\cos (\pi \alpha / 2 h)}, \\
& F_{2}\left(\frac{\alpha}{h}\right)=\frac{\sqrt{(2 h / \pi \alpha) \tan (\pi \alpha / 2 h)}\left[0.923+0.199(1-\sin (\pi \alpha / 2 h))^{4}\right]}{\cos (\pi \alpha / 2 h)} .
\end{aligned}
$$

The diagrams of flexibilities of the cracked shaft with respect to the cracked depth ratio are shown in Figure 4. The figures demonstrate that the flexibility of the cracked rotor increases in both the weak and strong crack direction. Obviously, flexibility in the weak crack direction grows more rapidly than the strong one. And the concaved up curve means that the influence of larger crack depth ratio appears to be more intense than slighter one. Coincidentally, the calculation show that when the crack depth ratio $a / D>0.5$, compared with the diagonal elements, effects of nondiagonal elements of dynamic behavior of the rotor system is relatively small just as the results of experimental analysis in $[15,16,18,19]$.
4.1. Transient Response. Setting constant acceleration strategy, the angular rotation function of time $t$ can be indicated as $\theta(t)=\int(\omega(t)) d t+C=(1 / 2) A t^{2}+B t+C$, where $A$ is the angular acceleration of the rotor, $B$ is the initial rotation speed, and $C$ is the initial angular position of the shaft. The static response with different initial conditions can be obtained by using Runge-Kutta numerical integration of the motion equations. The nondimensional unbalance response of the Jeffcott rotor with different mass eccentricity ratio, $\varepsilon=0.021 ; 0.001$, and crack depth ratio, $a / D=0.45 ; 0$, and unbalanced orientation angle, $\beta=\pi / 2$, is listed in Figure 5. 


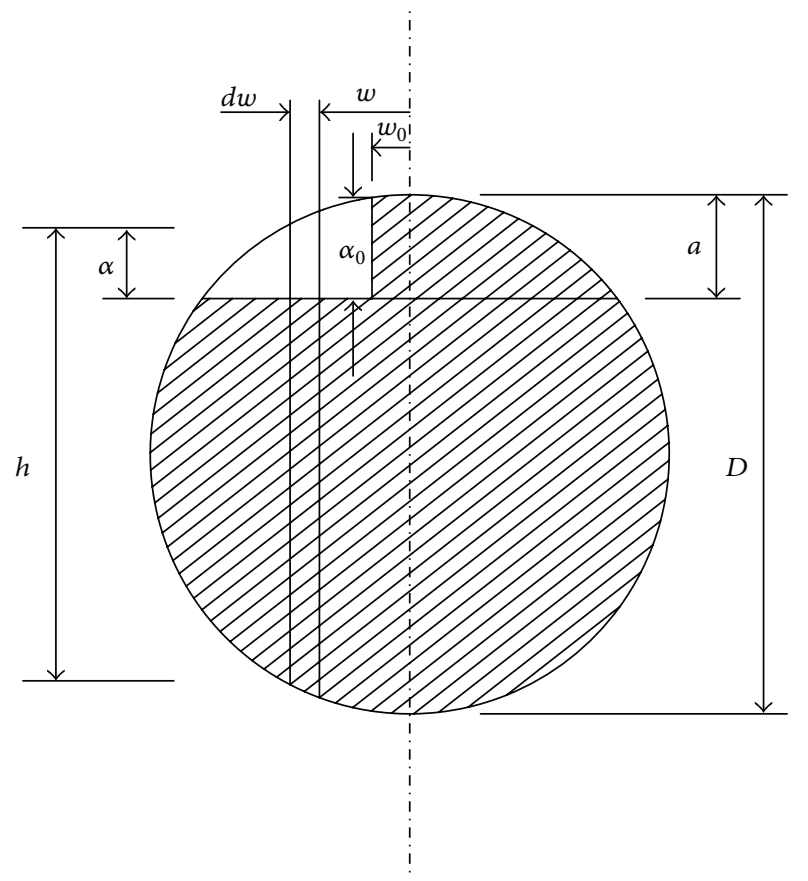

FIgURE 3: Cross section at crack location [27].

The responses of the rotating rotors with breathing crack in higher unbalance level exhibit the extreme instability as they approach the critical region comparing with the uncracked ones with lower unbalance level. The diagrams also show that both the responses in vertical and horizontal direction of rotor system each $t$ near their peak as they approach the critical speed. And then, due to the critical point excitation, the large amplitude free vibration with its natural frequency is not completely attenuated. The transient forced vibration synthesis causes the oscillation amplitude decreasing regularly. Due to the crack breathing, the peaks of the crackeds rotor are slightly later than the critical speed of the system. The critical resonance region near the critical speed of cracked rotors appears to be wider than the uncracked ones, and the amplitude of the responses of rotors with higher unbalance level increases much more rapidly than the ones with lower unbalance level. The orbital view of the cracked rotor with higher unbalance level deviates from the elliptic orbital in the vertical direction (Figure 5(b)). This interesting phenomenon demonstrates the compound influence of breathing crack and higher mass eccentricity on the nonlinear response of the rotating rotor as the rotation speeded up. In the supercritical region, there are basically three resonance peaks regions for the cracked rotor, which are the parametric resonances at $(3 / 2) \omega_{0}, 2 \omega_{0},(5 / 2) \omega_{0}$. Each peak is also later than the steady-state operation. As the rotation speeding up, resonance peaks still exist. But the magnitude of the peak is decreasing. And the higher the rotational speed, the more rapidly the resonance peak decreasing (Figures 5(e) and 5(f)). The transient responses of the periodic time-dependent rotor with varying parameters of the motion equations are generated with highly compliance with the actual experimental rotors.
Logarithmic amplitude spectrum comparison diagrams with respect to the dimensionless compound responses signal samples of cracked rotor and uncracked rotor near its critical speed with different mass eccentricity, $\varepsilon=0.001 ; 0.021 ; 0.042 ; 0.059$, are listed by FFT (Figure 6). Figure 6 shows that the mass eccentricity affects the amplitudes of the bending vibration of rotating rotors both cracked and uncracked significantly. The compound response of uncracked rotor rises smoothly near the critical speed with lower unbalance level (Figures 6(a) (A); 6(b) (A)), and the vibration has peaks of subcritical resonance and supercritical resonance at higher unbalance level (Figures 6(c) (A); 6(d) (A)). Contrary to the uncracked rotor, the response of cracked rotors is no longer smooth in both subcritical region and supercritical region not only for the higher unbalance level (Figures 6(c) (B); 6(d) (B)) but also for the lower unbalance level (Figures 6(a) (B); 6(b) (B)). The cracked rotors display abundant forms of nonlinear behavior. Near the critical region, the response of cracked rotor oscillates and does not have definite diagnostic speed, but a critical resonance region. There are subharmonic resonances in subcritical and supercritical region.

The cracked rotor system exhibits strong nonlinear motion due to the opening and closing crack. The response of the system in general is periodic motion, appears to be the double period motions in some speed, and also often appears to be harmonic components. When the crack depth is deep, there will be many nonlinear responses, such as double periodic motion, quasiperiodic motion, chaotic motion, Hopf bifurcation, and period doubling bifurcation $[4,5,18,19]$. The transverse vibration response appeared in the harmonic components which is obvious, such as 2-, 3-, and 4-octave frequency doubling and 5 octaves, and in a certain speed, there will be $3 / 2$ frequency.

4.2. Stability Analyze. Owning to the emergences of the crack, the stiffness of the rotor in two axes is not equal. In the preceding discussion, we know that the stiffness of the cracked rotor in two axes can be calculated by the stiffness of the uncracked rotor and crack depth ratio. Cracked rotor coupled with mass eccentricity may excite abundance unstable behavior and bifurcations during the horizontal rotating motion. Stability and bifurcations of a rotating gravity dominated cracked rotor with varied mass eccentricity are studied by the presented numerical method of computing eigenvalues of Floquet matrix based on the disturbing response. With the mass eccentricity $\varepsilon$ increased from 0 to 0.075 as the rotation speed ratio $p=\omega / \omega_{0}$ varied from 0.02 to 2.8 , the influence of the mass eccentricity on the stability of the dynamic system of cracked rotor is presented as a stability diagrams in the parameter plane in Figure 7(b) with parameters crack depth ratio $a / D=0.45$, unbalance orientation angel $\beta=\pi / 2$, and damping ratio, $\zeta=0.01$. And the stability analyst for uncracked rotor is also demonstrated as the comparisons to show the differences of influence of mass eccentricity on the uncracked rotor and cracked one in Figure 7(a).

For the uncracked rotor system, the unstable region (dark strips) around dimensionless rotation speed ratio appears 


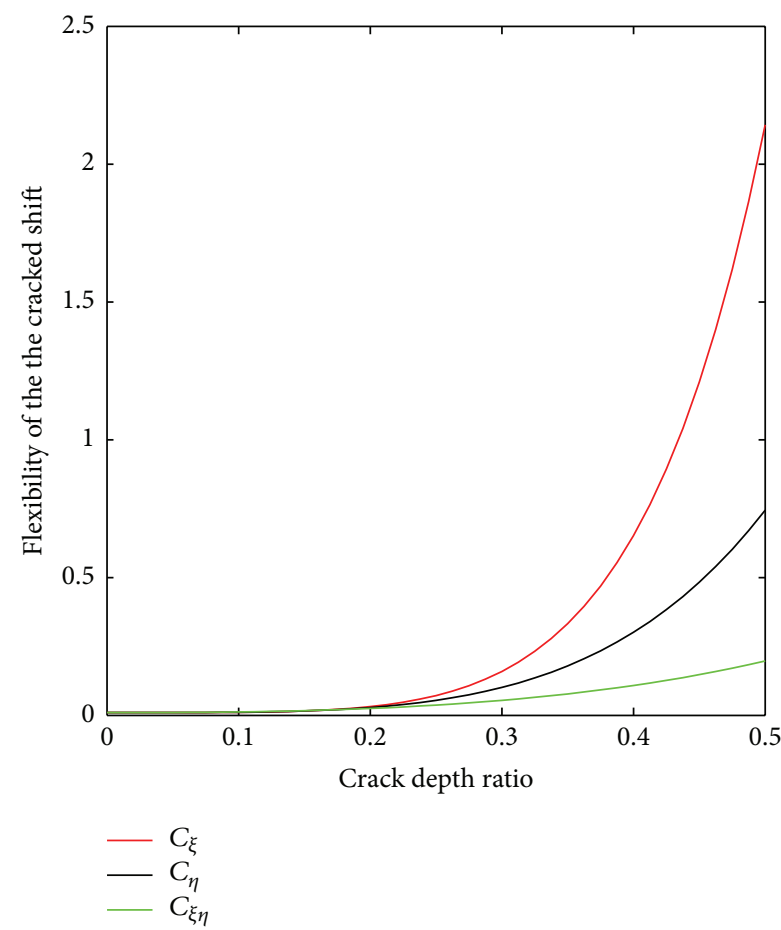

(a) Crack depth ratio from 0 to 0.4

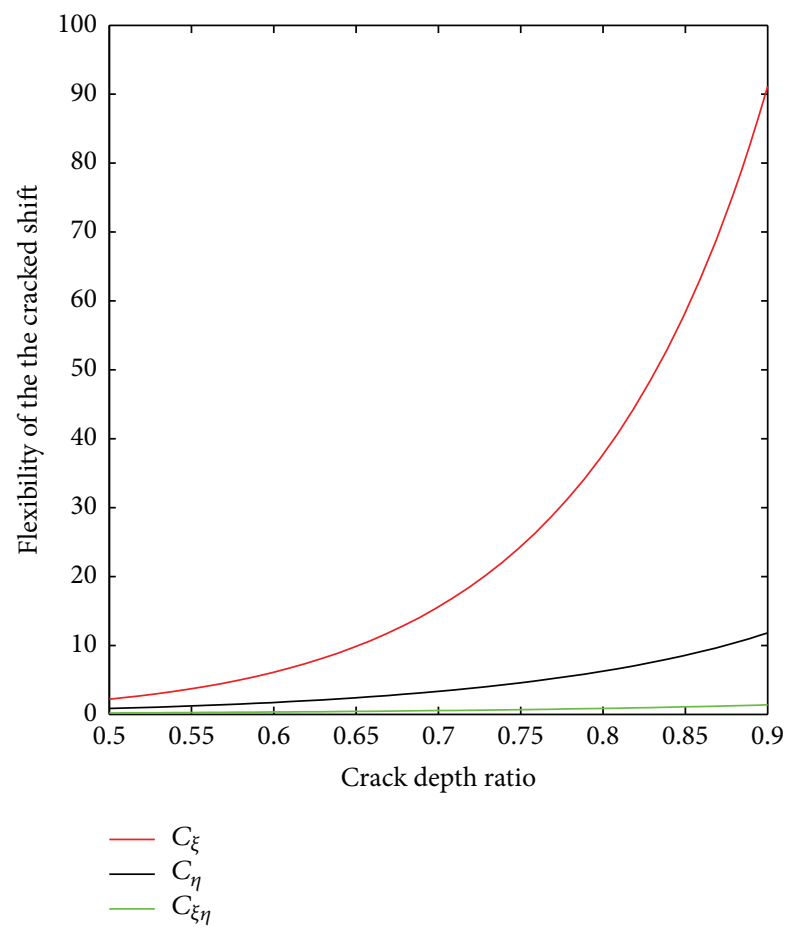

(b) Crack depth ratio from 0.4 to 0.8

FIgURE 4: The flexibility-crack depth ratio curve.

on the resonance region with the unbalance level of mass eccentricity larger than $\varepsilon=0.0054$, which means that the uncracked rotor can keep stable to pass through its resonance as the mass eccentricity less than $\varepsilon=0.0054$. This result coincides with the orbital tube plots of the responses of uncracked rotating rotor with minimal mass eccentricity in Figure 5. It is also observed from Figure 7(a) that the uncracked rotor system has unstable regions as the mass eccentricity becomes greater enough. Due to the overwhelmed perturbation caused by the high level of unbalance with mass eccentricity $(\varepsilon \geq$ 0.051 ) which is far beyond the attraction region of the system asymptotically stable zone, the uncracked rotor system which normally has been considered have no nonlinear effect transforms to be a periodic excitation system and begins to produce the nonlinear phenomenon not only nearby the first harmonic resonance but also in the subharmonic and superharmonic regions. The gradually expanded unstable zone around the resonance as the mass eccentricity growing testifies that the uncracked rotor with large mass eccentricity may also burst the abundant unbalance motion near its resonance $[2,12,15,17,26]$.

For the cracked rotor system, the unstable regions (dark strips) are arisen around dimensionless rotation speed ratio $p=\omega / \omega_{0}=\ldots ; 4 / 5 ; 3 / 2 ; 2 ; 12 / 5 ; \ldots$ even in case of nonmass eccentricity and expand gradually with respect to the increasing mass eccentricity ratio $[3,4]$. The resonance of the cracked rotor will occur earlier than the uncracked one $[3,5-$ $7,9]$. So as the speed ratio approaches to be $p=1$, there are no unstable strips between the first harmonic unstable and the second one until the mass eccentricity ratio exceeding 0.057 .
The result shows that the instable motion in the resonance region of the rotating cracked shaft which arose from the mass eccentricity is offset by the nonlinear breathing of the crack as the mass eccentricity no more than $\varepsilon=0.057$ and the cracked rotor system can even hardly keep its stable motion in a quit wide speed range around the first harmonic resonance and sub harmonic resonance with high level of unbalance degree shaft (i.e., the mass eccentricity ratio exceeding 0.057 ). These appearances are in agreement with the findings of previous works of Han and Chu [17] and are also consistent with the researches of Patel and Darpe [19].

The unstable regions of both uncracked and cracked rotors at relatively low rotation speed (less than $(1 / 2)\left(\omega_{0}\right)$ ) and high rotation speed (larger than $3 / 2 *\left(\omega_{0}\right)$ ) are also observed merely as the system with comparatively high mass eccentricity ratio. The unstable region (dark strips) for uncracked rotor in subharmonic and superharmonic regions emerges in case of comparatively higher unbalance level $(\varepsilon>$ 0.051 and $\varepsilon>0.07)$ than the cracked rotor $(\varepsilon>0.003$ and $\varepsilon>0.021$ ), which means that the cracked shaft can be distinct from the uncracked one by their unstable motion in subharmonic resonance given the mass eccentricity $\varepsilon<0.051$ and in superharmonic as the mass eccentricity $\varepsilon<0.07$. However, the instable motion in the resonance region of the rotating cracked shaft which arose from the mass eccentricity is offset by the nonlinear breathing of the crack as the mass eccentricity no more than $\varepsilon=0.057$.

Moreover, the unstable region for cracked rotor near dimensionless rotation speed ratio $p=\omega / \omega_{0}=2 ; 12 / 5$ just occurs as the mass eccentricity ratio exceeding 0.021 , 


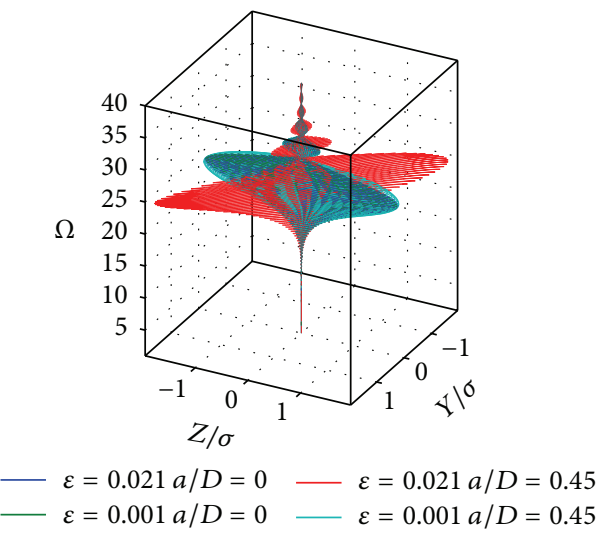

(a) Orbital tube

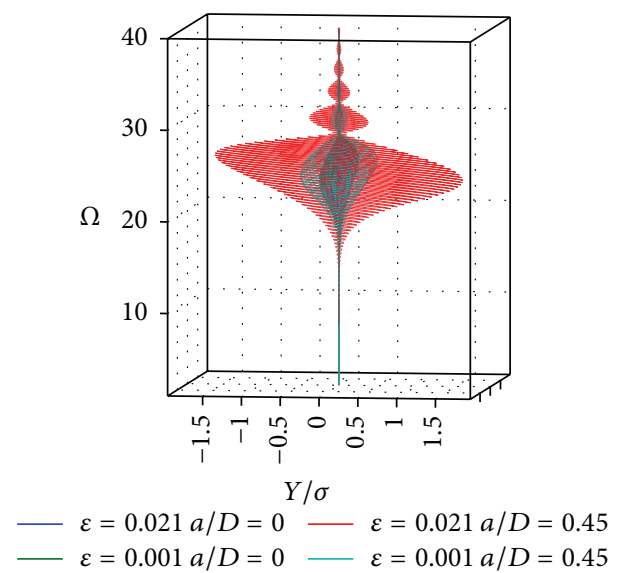

(c) Deceleration view for $Y$-direction

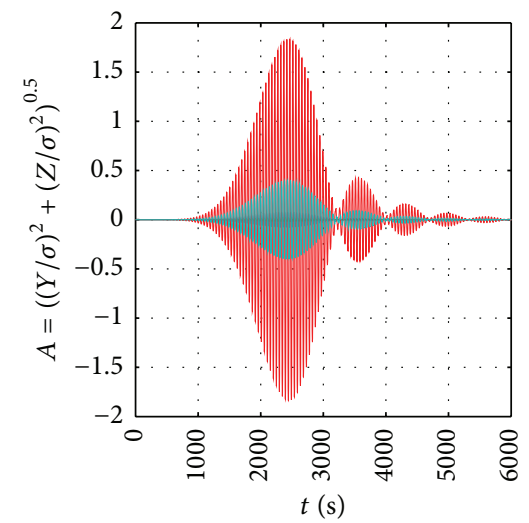

$\begin{aligned} \varepsilon & =0.021 a / D=0 \\ \varepsilon & =0.001 a / D=0 \\ \varepsilon & =0.021 a / D=0.45 \\ \varepsilon & =0.001 a / D=0.45\end{aligned}$

(e) Compound response

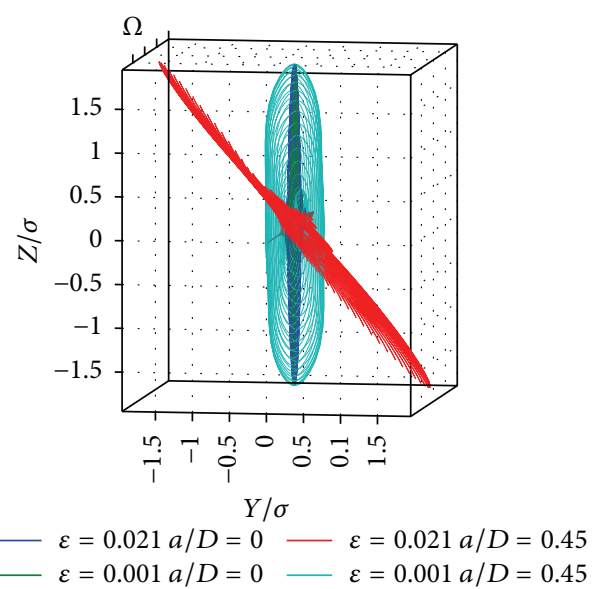

(b) Orbital view

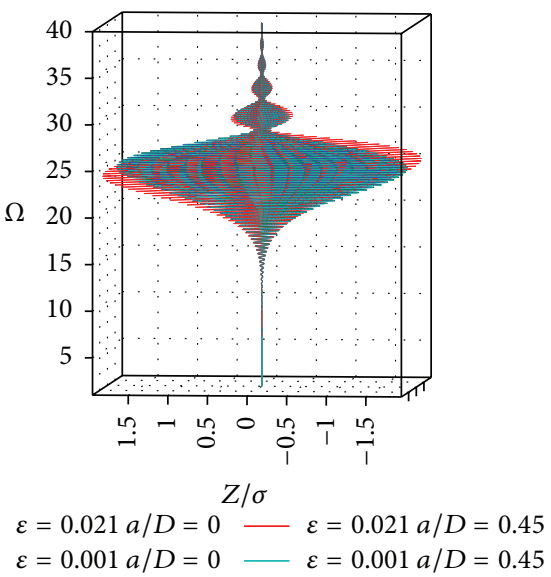

(d) Deceleration view for $Z$-direction

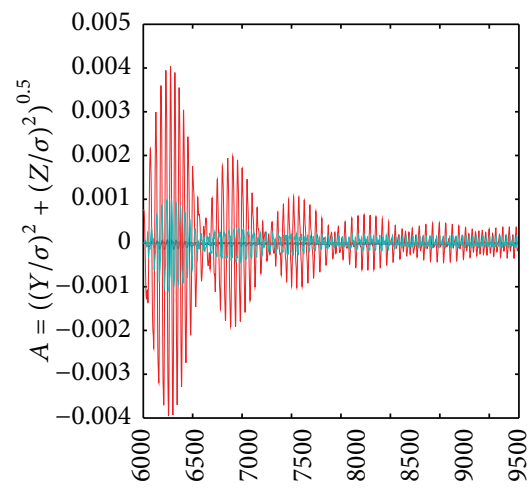

$t(\mathrm{~s})$

$\begin{aligned} \varepsilon & =0.021 a / D=0 \quad-\varepsilon=0.021 a / D=0.45 \\ \varepsilon & =0.001 a / D=0 \quad-\varepsilon=0.001 a / D=0.45\end{aligned}$

(f) Compound response

FIGURE 5: Nondimensional unbalance response of the Jeffcott rotor with a breathing crack with different mass eccentricity ratio $\varepsilon=$ $0.021 ; 0.001$, crack depth ratio $a / D=0.45 ; 0$, and unbalanced orientation angle $\beta=\pi / 2$ : (a) orbital tube; (b) orbital view; (c) deceleration view for $Y$-direction; (d) deceleration view for $Z$-direction; (e) compound response $A=\sqrt{(Y / \sigma)^{2}+(Z / \sigma)^{2}}$ within time interval from 0 sec to $6000 \mathrm{sec}$; (f) compound response $A=\sqrt{(Y / \sigma)^{2}+(Z / \sigma)^{2}}$ within time interval from 6000 sec to $9800 \mathrm{sec}$. 

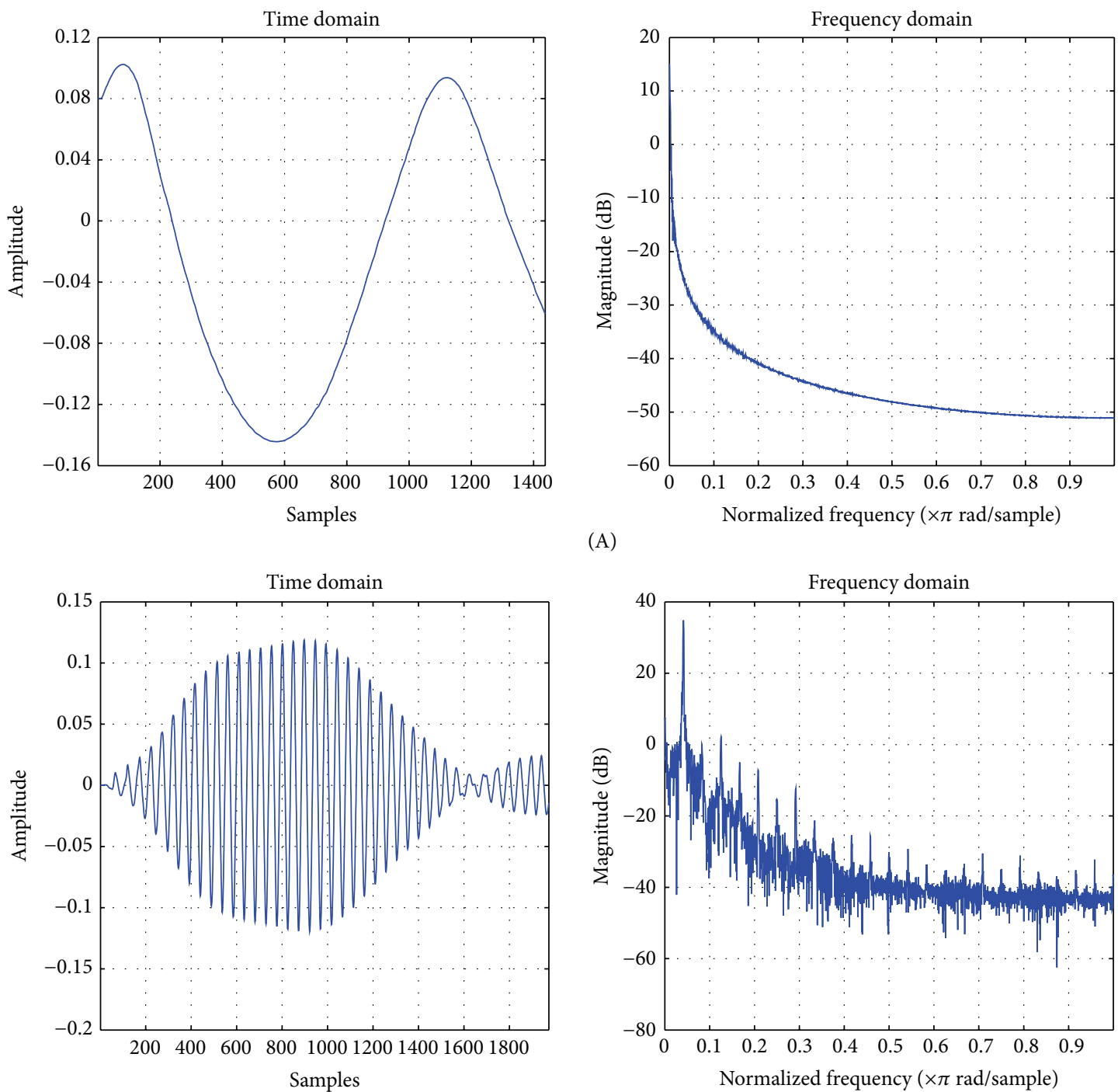

(A)

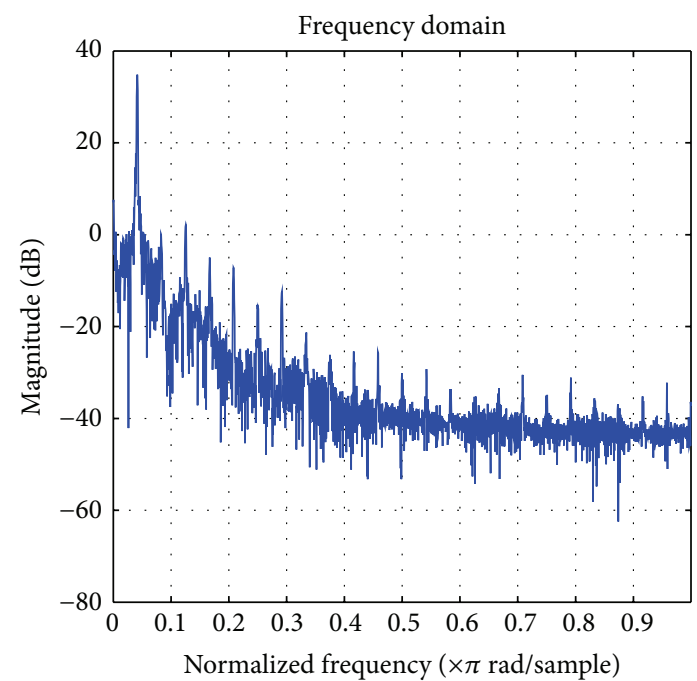

(B)

(a)
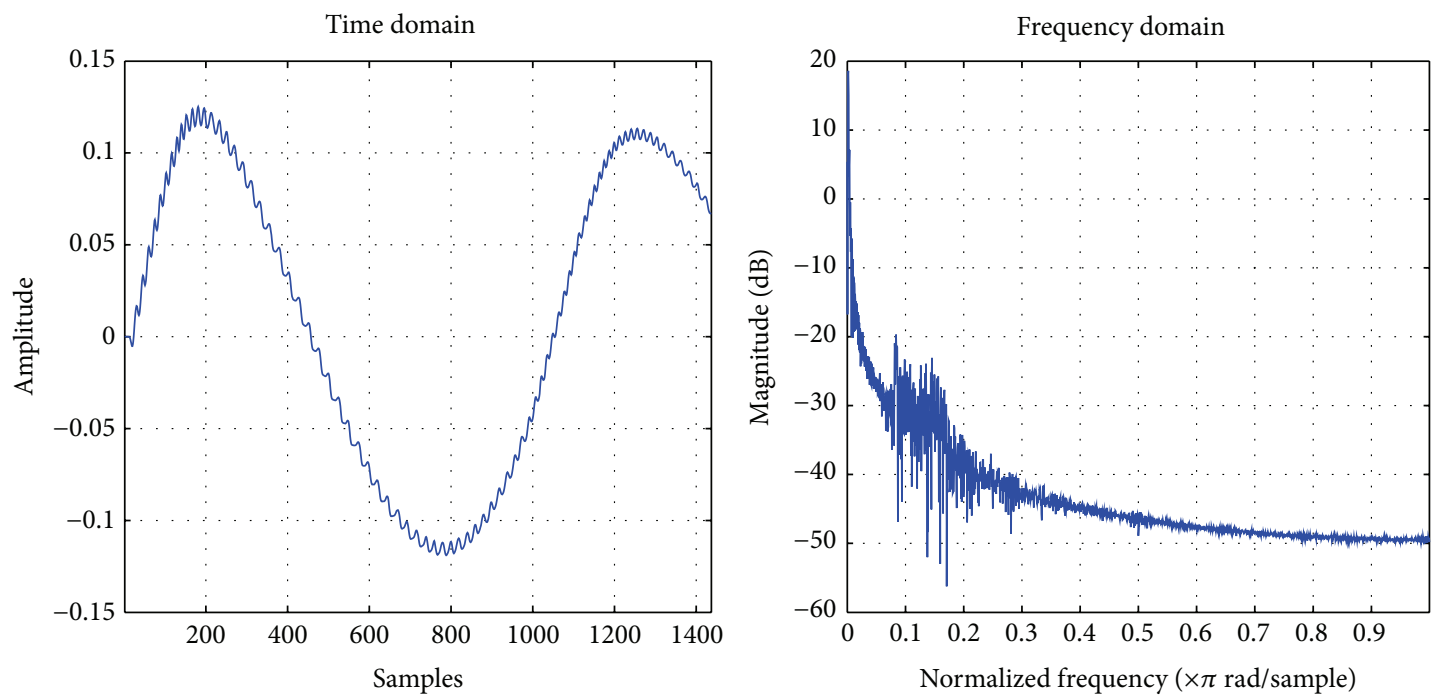

(A)

Figure 6: Continued. 

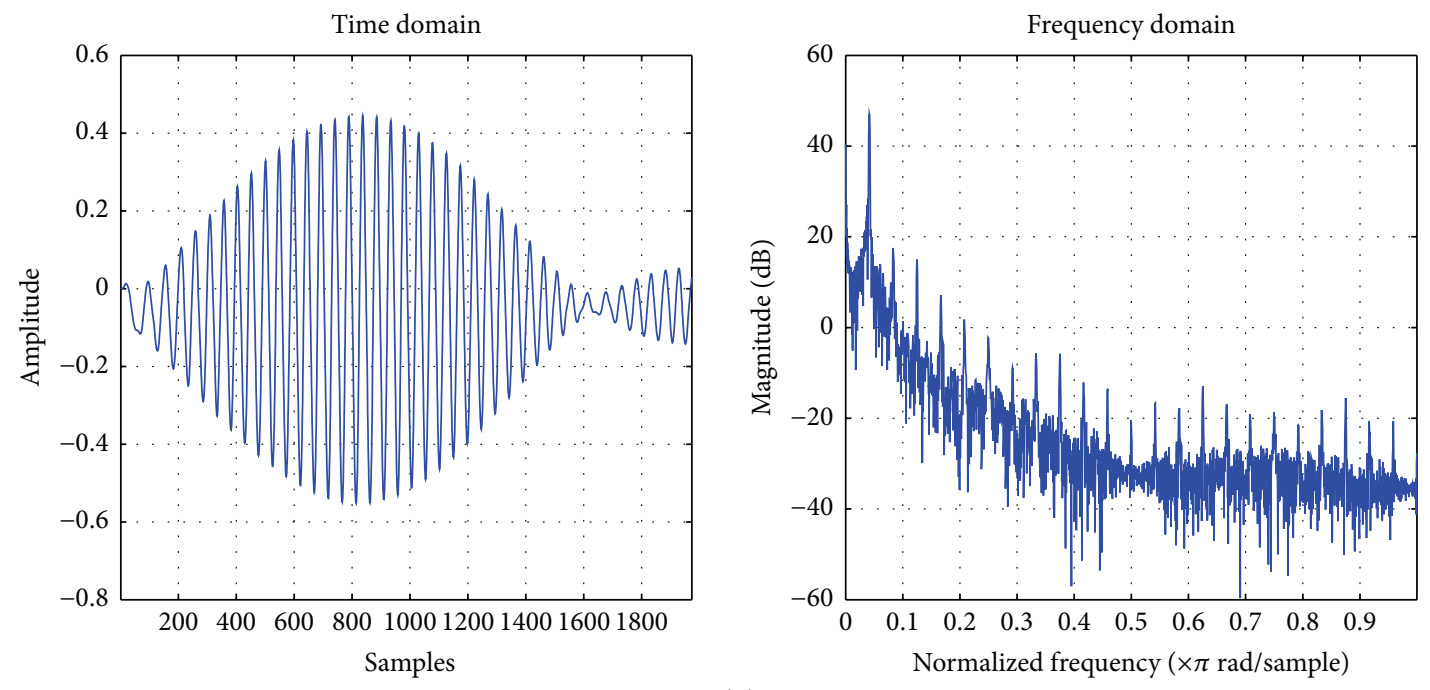

(B)

(b)
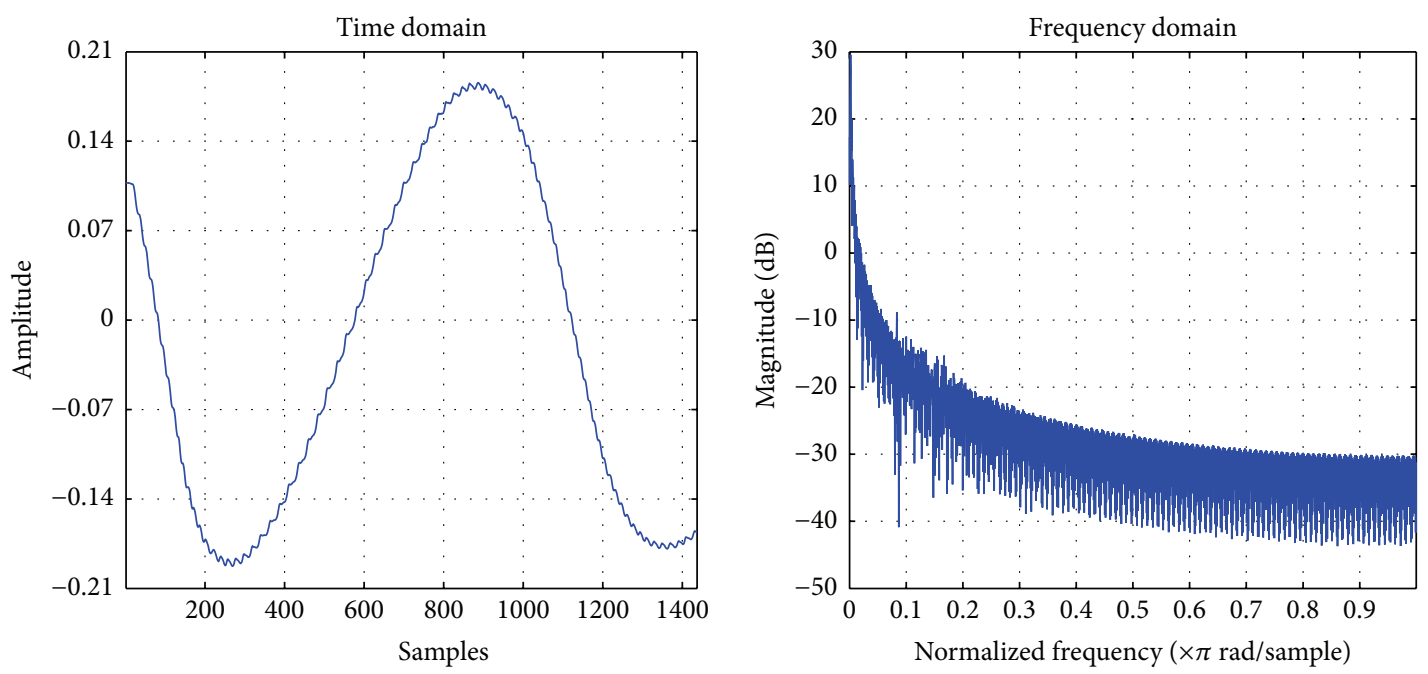

(A)
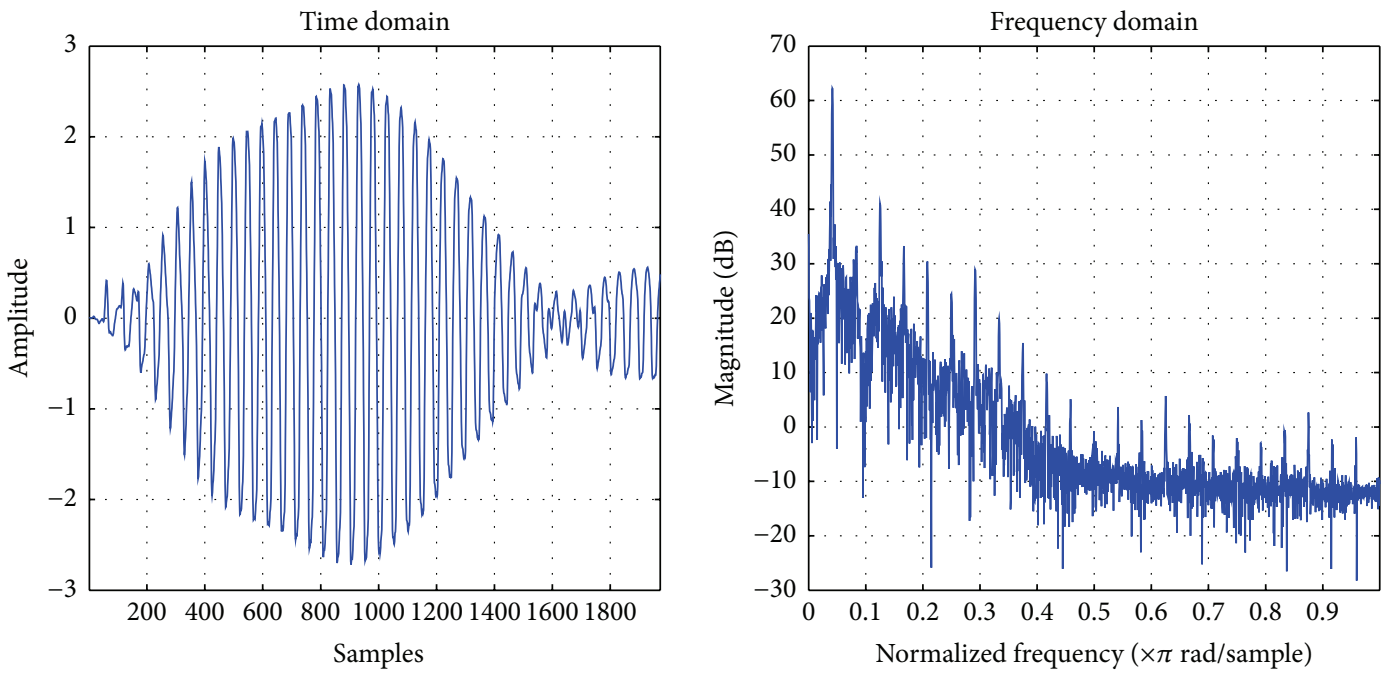

(B)

(c)

FIgure 6: Continued. 

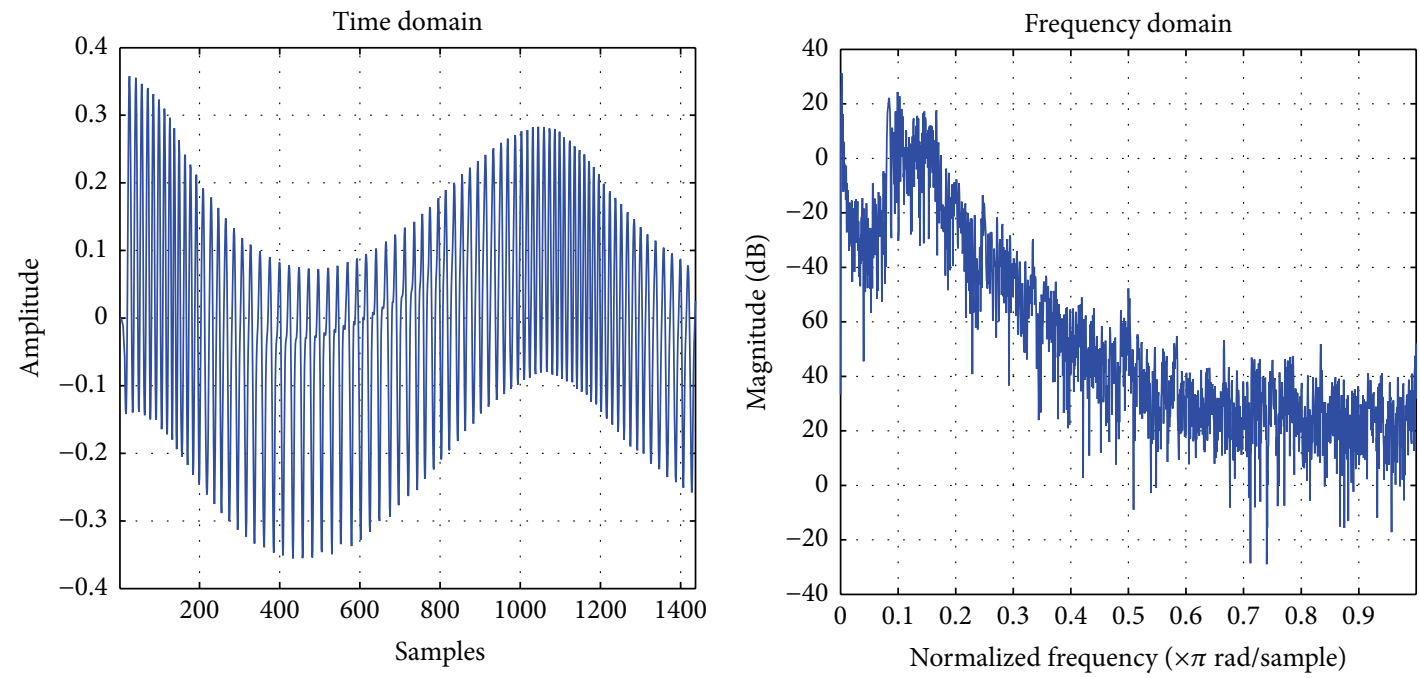

(A)
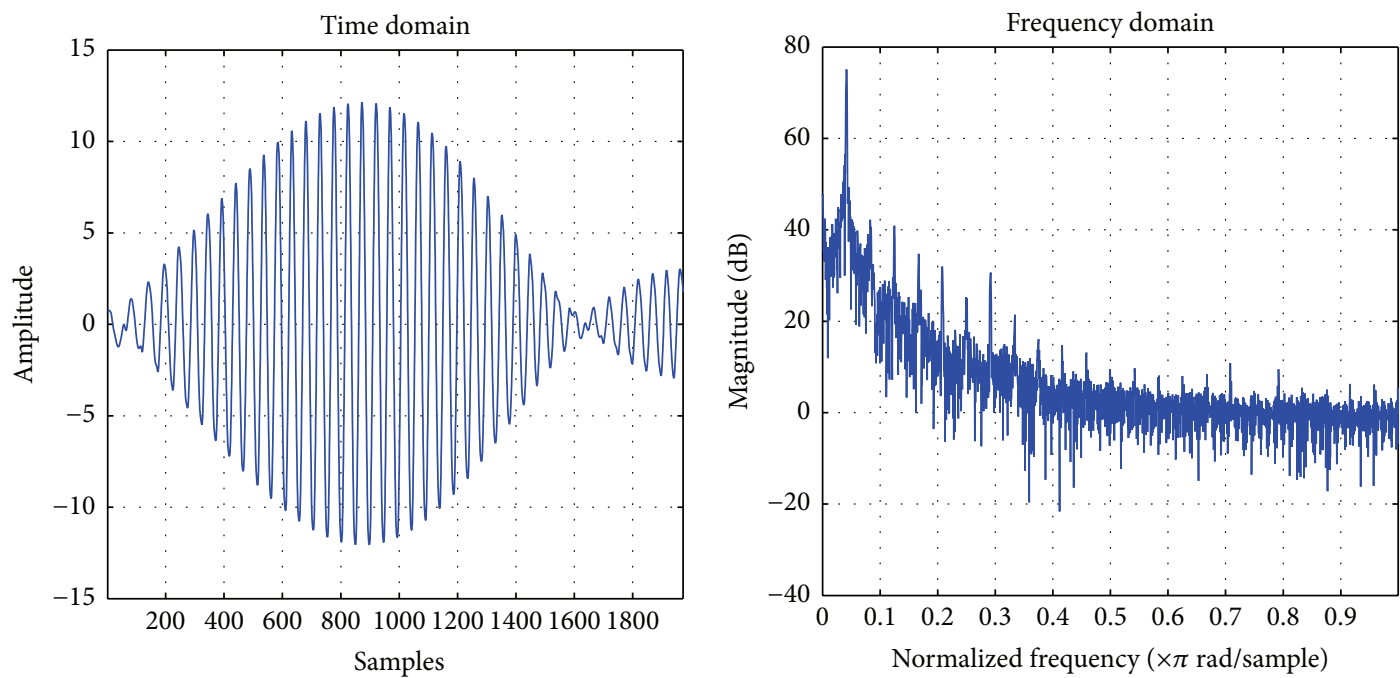

(B)

(d)

FIGURE 6: Logarithmic amplitude spectrum comparisons of cracked rotor and uncracked rotor near its critical speed with different mass eccentricity: (a) $\varepsilon=0.001$; (b) $\varepsilon=0.021$; (c) $\varepsilon=0.042$; (d) $\varepsilon=0.059$, (A) for uncracked rotor; (B) for cracked rotor with parameters $a / D=0.45, \beta=\pi / 2$.

while the unstable region near dimensionless rotation speed ratio $p=\omega / \omega_{0}=4 / 5 ; 2 / 3$ originates even the mass eccentricity ratio approaches to non. However, the width of the unstable zones for the cracked rotor around $p=\omega / \omega_{0}=1$ seems to be reduced slightly than the superharmonic unstable zones. For the mass eccentricity less than 0.021 , the unstable region (dark strips) for the cracked rotor disappears at the superharmonic resonances area as $p>1.6$. Particularly for the trivial unbalance degree (i.e., the mass eccentricity less than 0.003), the unstable region shrinks to be quite narrow strips, which means the cracked rotor system can keep its stability of the rotation motion with high stable degree as the rotation speed fleeting through the sharp unstable region near the subharmonic and harmonic resonance $[13,18,19,25]$.
4.3. Bifurcations at Boundary of the Stable Region near the Critical Rotation Speed. We use Floquet theory to analyze the bifurcations at the boundary points of the stable region near the critical rotation speed. The periodic solution is asymptotically stable if the unit circle in the complex plane encloses all the Floquet multipliers [9, 11]. The periodic solution is critical stable if there is at least one Floquet multiplier lies exactly on the unit circle and all the others inside the unit circle. The periodic solution is unstable if one of the Floquet multipliers lies outside the unit circle.

There are basically three types bifurcations for the periodical solutions for the breathing crack model with parameters $a / D=0.45, \beta=\pi / 2, \varepsilon=0.021$ shown in Figure 8. Periodic solutions generate saddle node bifurcations and instability when there is at least one Floquet multiplier going out the 


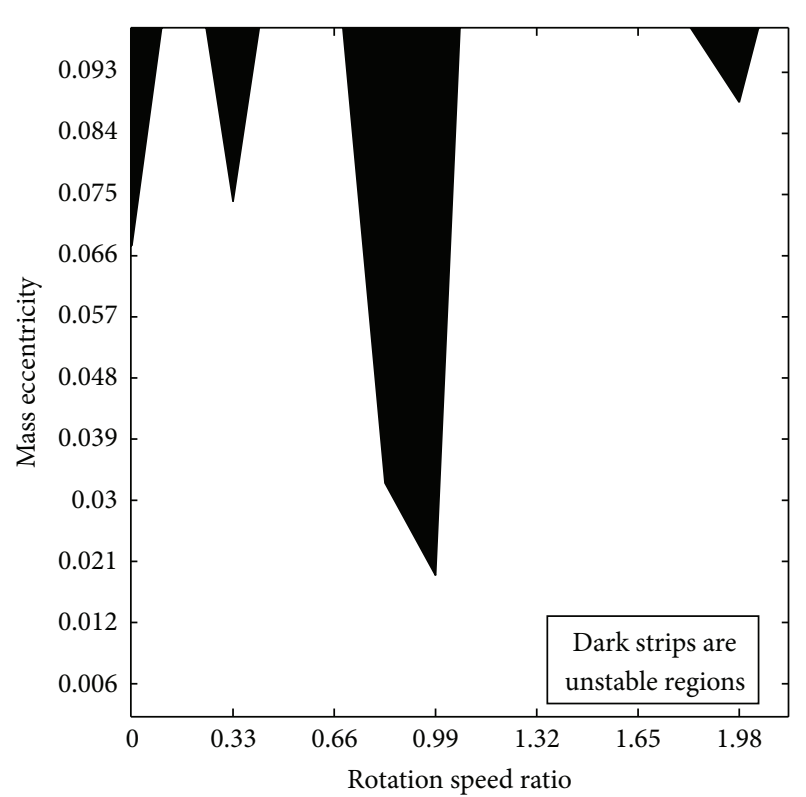

(a)

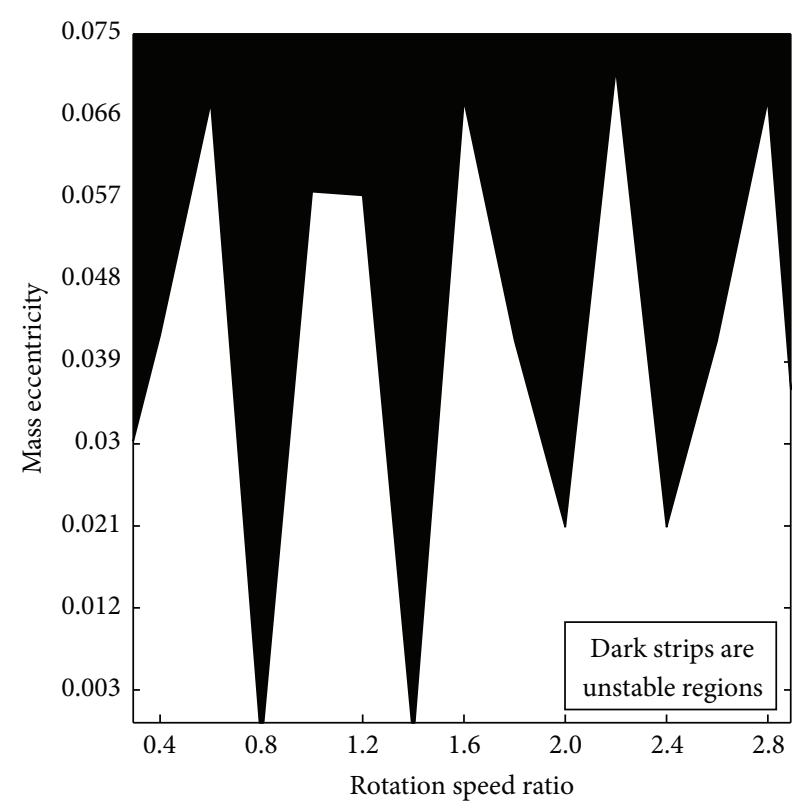

(b)

FIGURE 7: Stability diagrams with respect to the varied mass eccentricity with parameters for the speed up uncracked rotor (the dark zones are unstable regions and the white zones are stable regions) (a) with parameters: $a / D=0$, unbalance orientation angel $\beta=\pi / 2$, and damping ratio $\zeta=0.01$; and the cracked rotor $(b)$ : crack depth ratio $a / D=0.45$, unbalance orientation angel $\beta=\pi / 2$, and damping ratio $\zeta=0.01$.

unit circle through the point $(1,0)$ and others keeping inside the unit circle. Periodic solutions generate double period bifurcations when there is at least one Floquet multiplier going out the unit circle through the point $(-1,0)$ and others keeping inside the unit circle. Periodic solutions generate Hopf bifurcations and second Hopf bifurcations when there are a pair of conjugate Floquet multipliers going out the unit circle and others keeping inside the unit circle. Figure 8 shows that the probability statistics of the Floquet multiplier dots demonstrate higher densities near the point $(1,0)$ and the point $(-1,0)$ than others boundaries, which mean that the Hopf bifurcations are more exiguous than the other two kinds of bifurcations. This specific appearance is consistent with the results of Guo et al. in [5] and Patel and Darpe [19]. Due to the mass eccentricity, which makes the additional the disturbance, Hopf bifurcations exist inside the unstable zones as the rotating speed approach the critical rotation speed. This indicates that rotor with fatigue cracks (even the crack depth ratio is not extremely large) may excite abundance unstable behavior and bifurcations especially coupled with the disturbance of the mass eccentricity during the horizontal rotating motion.

4.3.1. Double Period Bifurcations. We study the bifurcation behaviors of the rotating cracked rotor with the parameters $a / D=0.45, \beta=\pi / 2$, and $\varepsilon=0.021$. According to Floquet theory, there are double period bifurcations when one of Floquet multiplier goes out the unit circle through the point $(-1,0)$. And the period of response will be double. We find that the response is periodic one motion at $p=$ 0.6015 (Figure 9(a)) while the response turns to be periodic two motion at $p=0.6018$ (Figure 9(b)). We evaluate the eigenvalues of the state transition matrix (Floquet matrix) and find one of the eigenvalue is -1.0018 , which mean there is a double period bifurcation. Another example is taken from $p=1.3570$ to $p=1.3573$ (Figures $9(\mathrm{c})$ and $9(\mathrm{~d})$ ). The motion bifurcates from period 4 to period 8 . One of the eigenvalue of the Floquet matrix is -1.0027 in this time which means that the double period bifurcation occurs here.

4.3.2. Saddle-Node Bifurcations. There are saddle-node bifurcations when one of Floquet multiplier go out the unit circle through the point $(1,0)$. And the system response bifurcates from a periodic motion to another periodic motion. The motion of rotor bifurcates from period 8 at $p=1.4576$ to period 6 at $p=1.4578$ (Figures $9(\mathrm{~g})$ and $9(\mathrm{~h})$ ). We evaluate the eigenvalues of the Floquet matrix and find an eigenvalue is 1.006, which means there is a saddle-node bifurcation here. Another saddle-node bifurcation can be find between $p=$ 1.3573 and $p=1.4576$ (Figures $9(\mathrm{f})$ and $9(\mathrm{~g})$ ). Although both of their motions are period 8 , their orbit plots are far different. We find the Floquet multiplier at 1.4257 is 0.9989 . It involves a saddle-node bifurcation there.

4.3.3. Hopf Bifurcations. From Floquet theory, the system response has Hopf bifurcations when there is a pair of conjugate Floquet multipliers being out the unit circle. And responses bifurcate from periodical motion to quasiperiod motion. We take the example of responses at $p=0.6529$ and $p=0.6531$ (Figures 9(c) and 9(d)), and the motion of rotor bifurcates from period 3 to quasiperiod. We evaluate the eigenvalues of the Floquet matrix and find that a pair of 


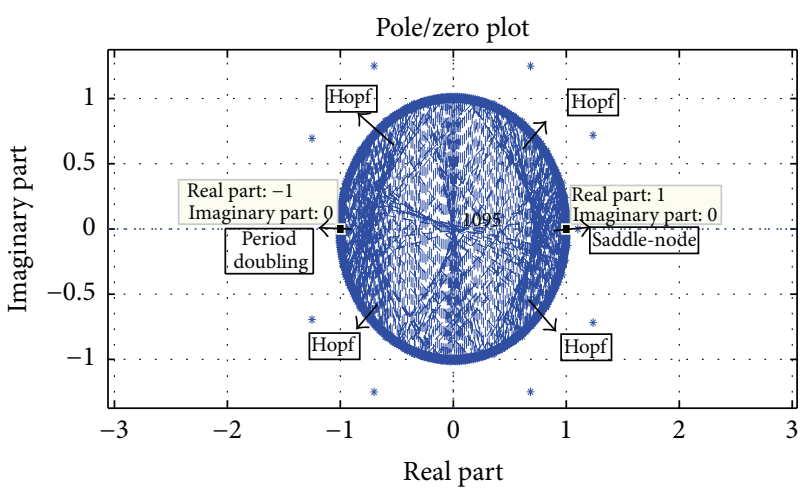

Figure 8: Three modes of bifurcations defined by Floquet multipliers through the unit circle on the complex plane.

complex eigenvalues is $0.5021+i 0.8680$, and its modulus is 1.0028 , which means a Hopf bifurcation there.

The bifurcation may happen to be chaotic (Figure $9(\mathrm{l})$ ) or quasiperiodic (Figures 9(j) and $9(\mathrm{k})$ ) or subharmonic motion (Figure 9(i)) from periodic motion once the rotation speed approaches the critical or subcritical speed.

Beside the abundant form of quasiperiodic motion (Figures $9(\mathrm{e}), 9(\mathrm{j})$, and $9(\mathrm{k})$ ), chaotic and quasiperiodic nature at some speed ratio are also observed in the rotating cracked rotor system (Figures $9(\mathrm{j})$ and $9(\mathrm{l})$ ).

The noted three kinds of bifurcations mainly bifurcate from periodic motion to chaotic or quasiperiodic motion, which are consisted with the researches of Guo et al. [5], Patel and Darpe [19], and Untaroiu et al. [4]. The motion of the cracked rotor system usually bifurcates from periodic motion to quasiperiodic motion at Hopf bifurcations. The dynamic response usually bifurcates from a kind of periodic motion to another periodic motion at saddle-node bifurcations. There also exists period doubling bifurcation at the boundary points of the stable region of the cracked rotor system.

Based on the numerical analyses of Floquet multipliers, we can detect the type of bifurcations of the transient response of the rotating cracked rotor system, which may provide an efficient quantitative methodology in the identification of cracks in rotating machinery and consequently undertake the visualized comparisons for the responses of the cracked rotor system at these bifurcations.

\section{Conclusions}

The study is proposed to give a feasible method to diagnose the stable and unstable regions of the rotating cracked rotor system and to investigate the bifurcations on boundary points based on the detailed numerical eigenvalues of the transition matrix which may provide the basis for monitoring and diagnosing fault of rotating machines. The motion of the gravity dominated rotating cracked rotor is described by the equations with the harmonic breathing crack function and the periodic time-dependent cross-coupled stiffness originated from the flexibility of the shaft in the direction of the crack and orthogonal to the crack direction. The transient response of the rotation motion of the cracked rotor is analyzed by the magnitude of the modulus of eigenvalues of Floquet matrix derived from the disturbing response.

By means of the numerical transition matrix approach, the influence of the varied mass eccentricity on the stability of the periodic time-dependent rotor with a transverse breathing crack is listed as a stability diagram in the parameter plane as the rotation speed up. Consisted with the presented frequency spectrum as the rotation speed approaching the critical rotation speed, the stability diagram shows that the cracked rotor system can hardly keep its stable motion around the first harmonic resonance and sub harmonic resonance with high level of unbalance degree shaft (i.e., the mass eccentricity ratio exceeding 0.057 ). However, for low level of unbalance degree shaft (i.e., the mass eccentricity ratio less than 0.003), the cracked rotor system can keep its stable motion with higher stability degree as the rotation speed fleeting through the sharp unstable region near the subharmonic and harmonic resonance. The difference between the instable motions of the cracked rotor and noncrack one excited by mass eccentricity is extremely distinguished inside the mass eccentricity range $\varepsilon<0.051$ at subharmonic resonance and $\varepsilon<0.07$ in superharmonic resonance. But at the first resonance region, the instable motion in the resonance region of the rotating cracked shaft which arose from the mass eccentricity is offset by the nonlinear breathing of the crack as the mass eccentricity no more than $\varepsilon=0.057$. These result may provide a way for crack detection and instability control of the heavy loading turbo-machinery system.

The detailed numerical eigenvalues of the transition matrix are applied to analyze the types of the bifurcations of the cracked rotor system. Three types of bifurcations are found and responses of the cracked rotor system at these bifurcations are presented to undertake the visualized comparisons. Distinct from the foregoing researches by Guo et al. [5] and Patel and Darpe [19], Hopf bifurcations are also found inside the unstable zones as the rotating speed approaches the critical rotation speed beside the period doubling bifurcations and saddle-node bifurcations. This indicates that rotor with fatigue cracks (even the crack depth ratio is not extremely large) may excite abundance unstable behavior and bifurcations especially coupled with the relatively high unbalance level of the mass eccentricity during the horizontal rotating motion. From simulation results, the jumping from one periodic to another periodic motion is observed at saddle-node bifurcation and the motion from period to quasiperiod at Hopf bifurcation is also presented. There also exists period doubling bifurcation at the boundary points of the stable region of the rotor system.

\section{Nomenclature}

$C_{\xi \eta}=C_{\eta \xi}:$ Coupling flexibility coefficient

$\widehat{k}_{\xi}$ : The stiffness of the cracked shaft in $\xi$

direction

$\widehat{k}_{\eta}: \quad$ The stiffness of the cracked shaft in $\eta$

$\Xi(\Omega)$ : Breathing function for the crack 


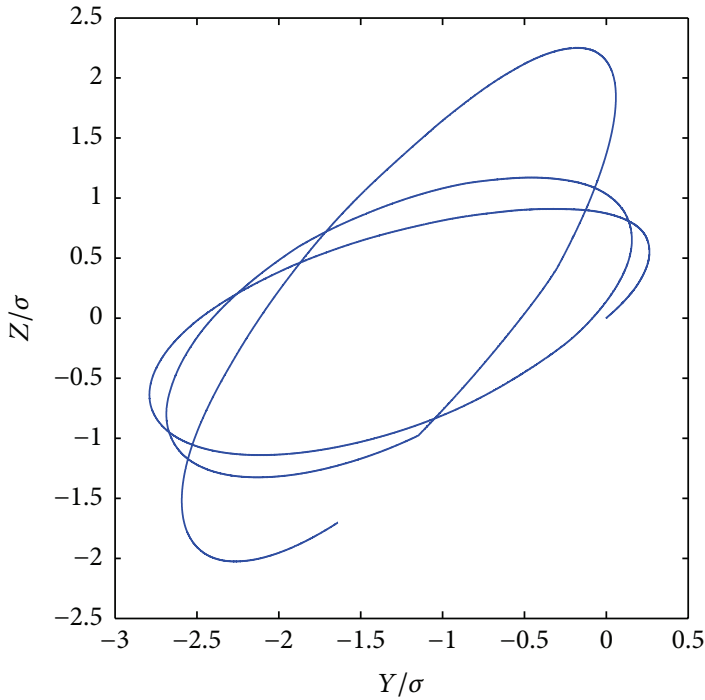

(a)

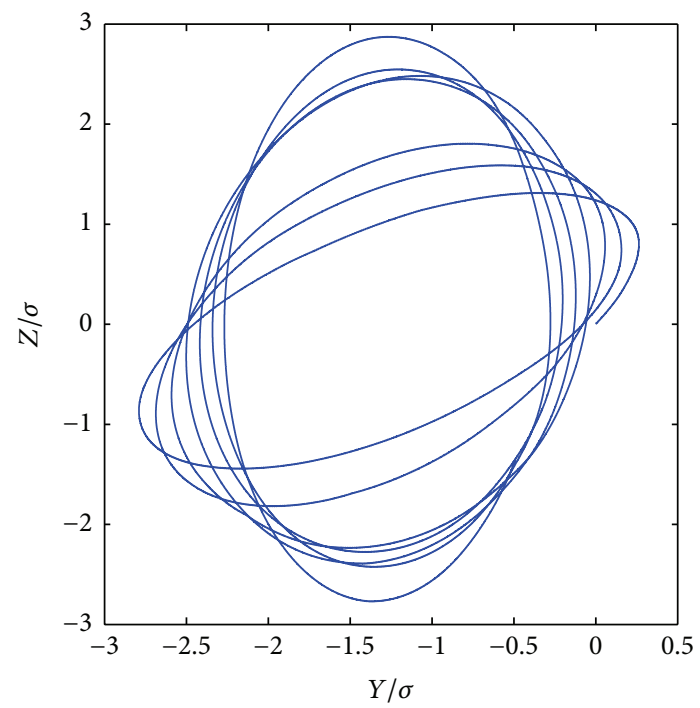

(c)

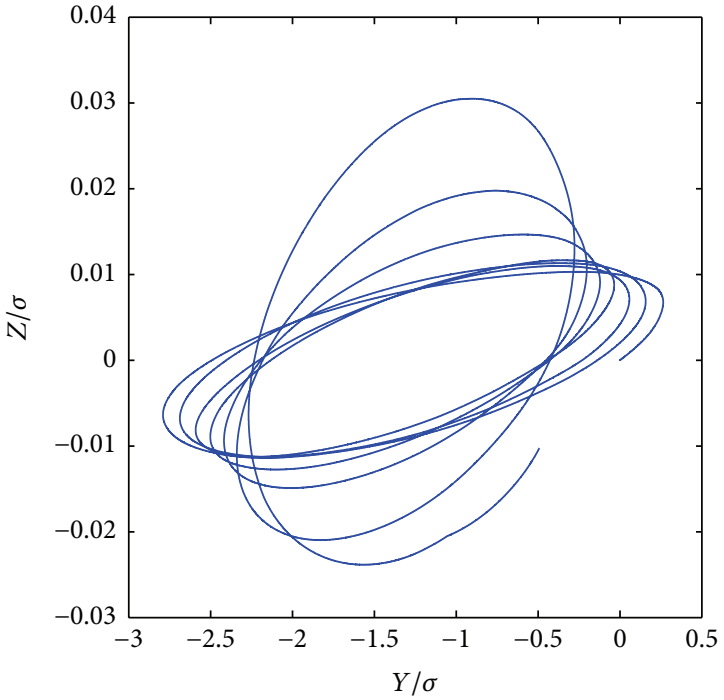

(e)

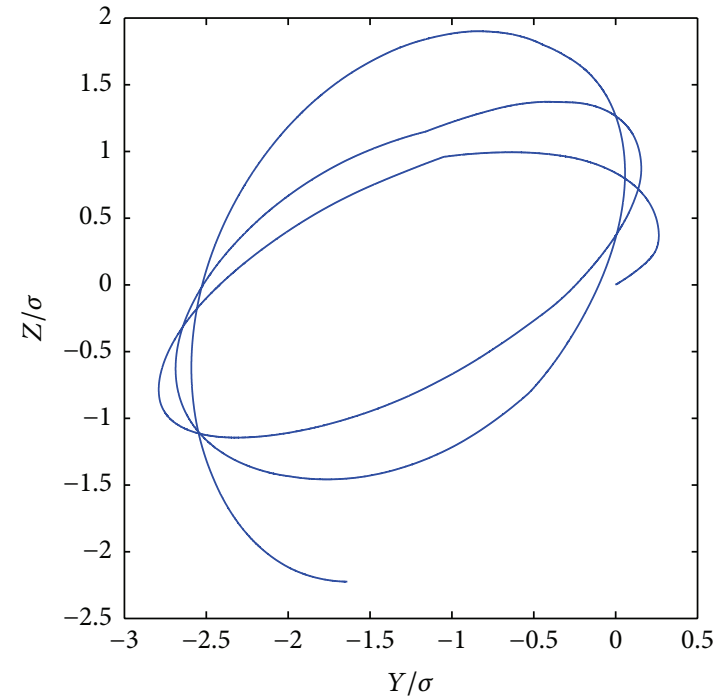

(b)

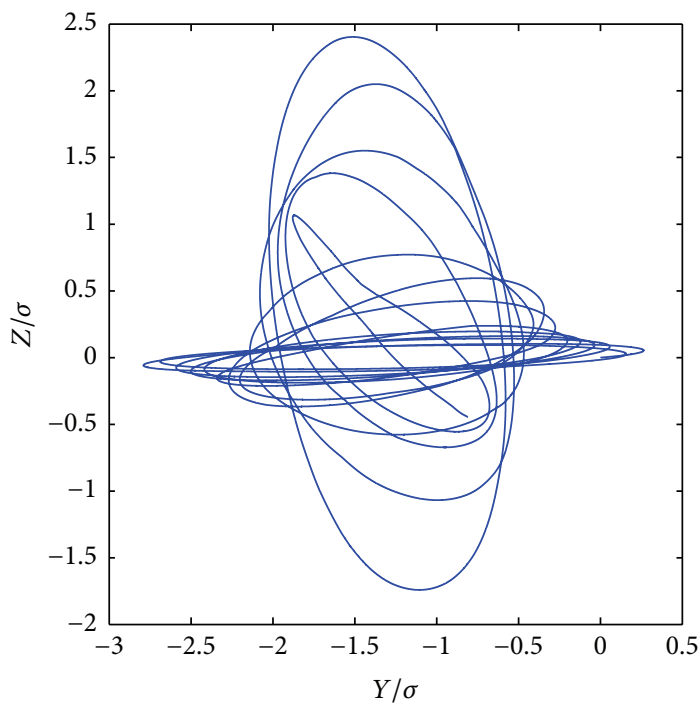

(d)

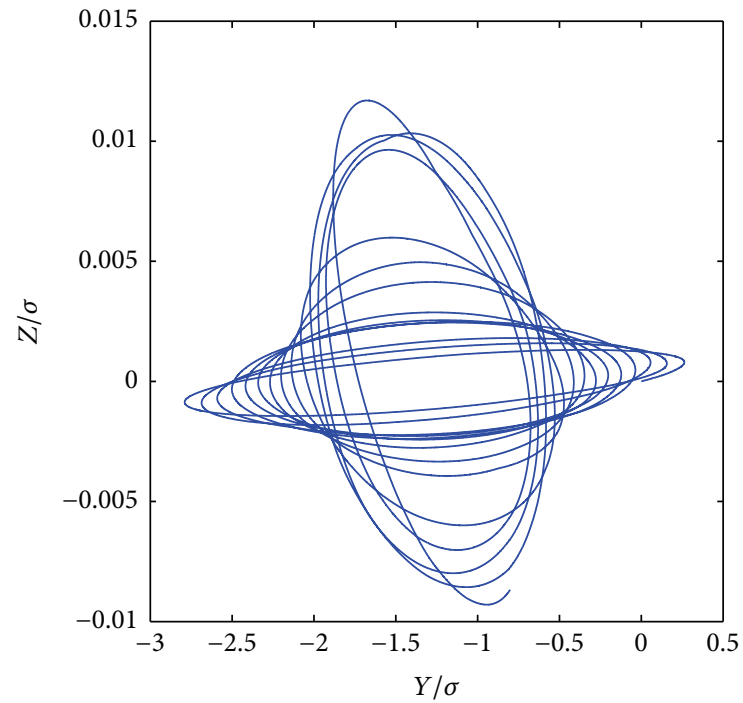

(f)

Figure 9: Continued. 


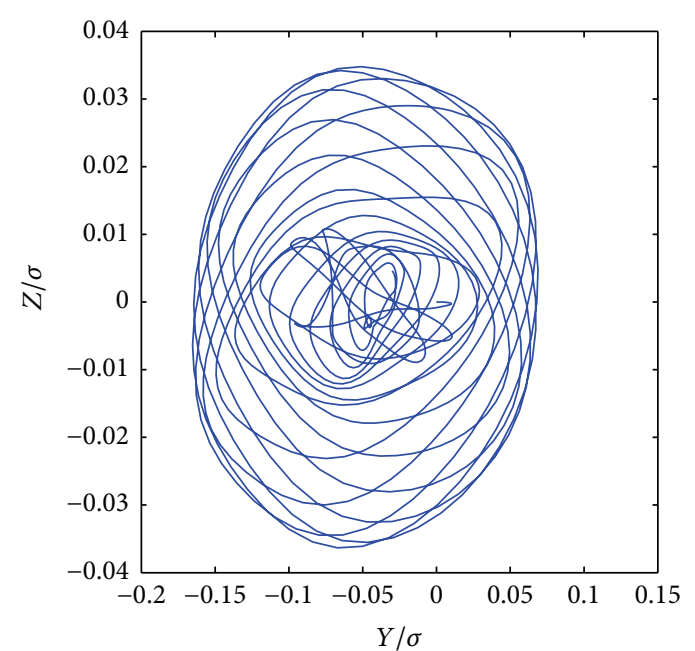

(g)

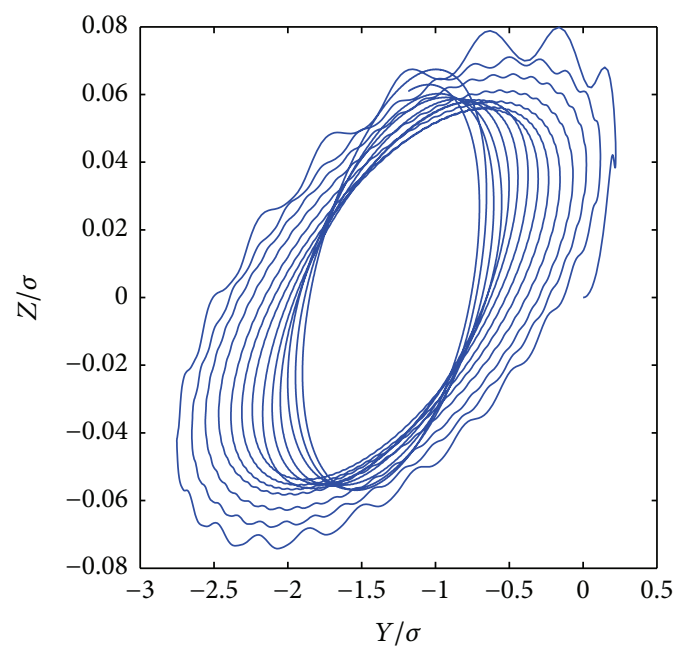

(i)

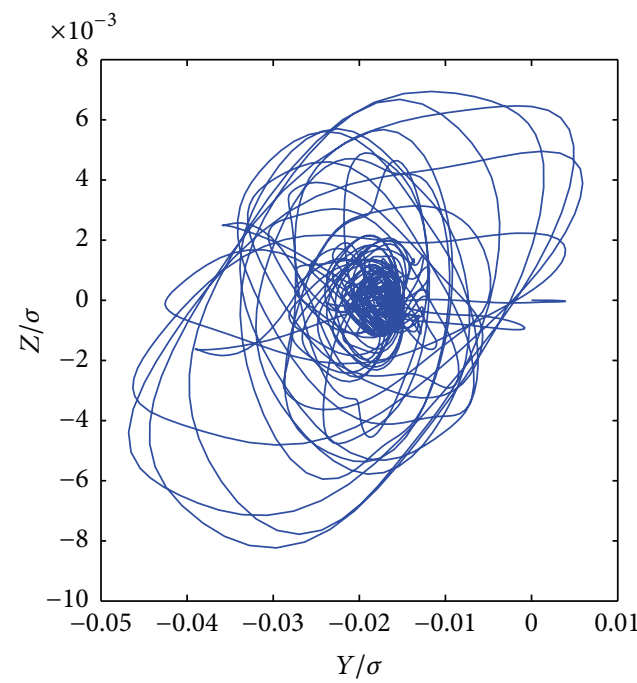

(k)

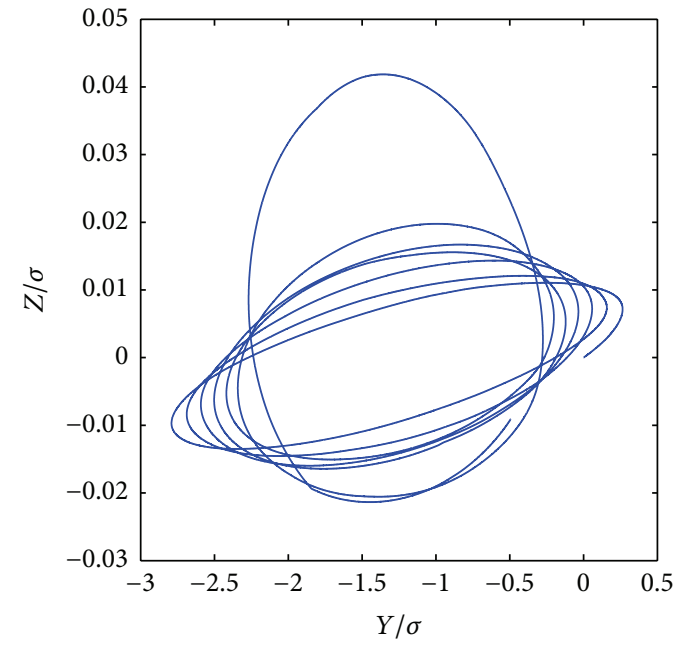

(h)

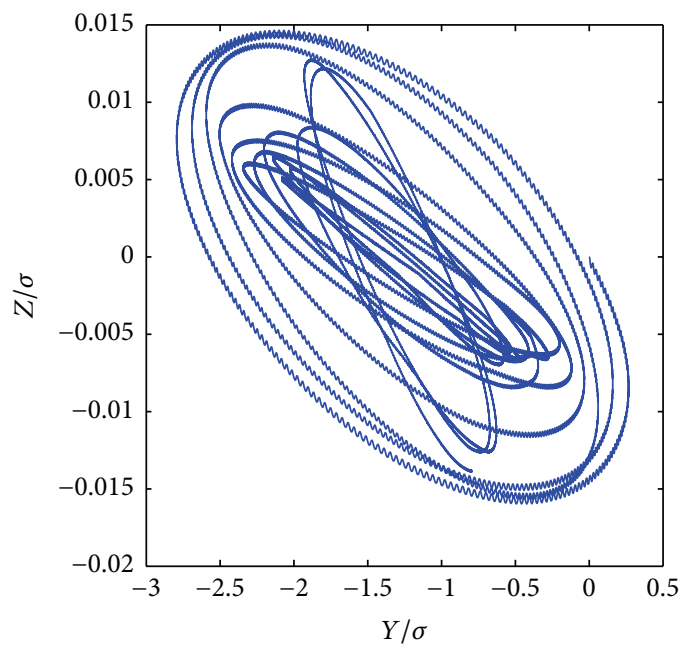

(j)

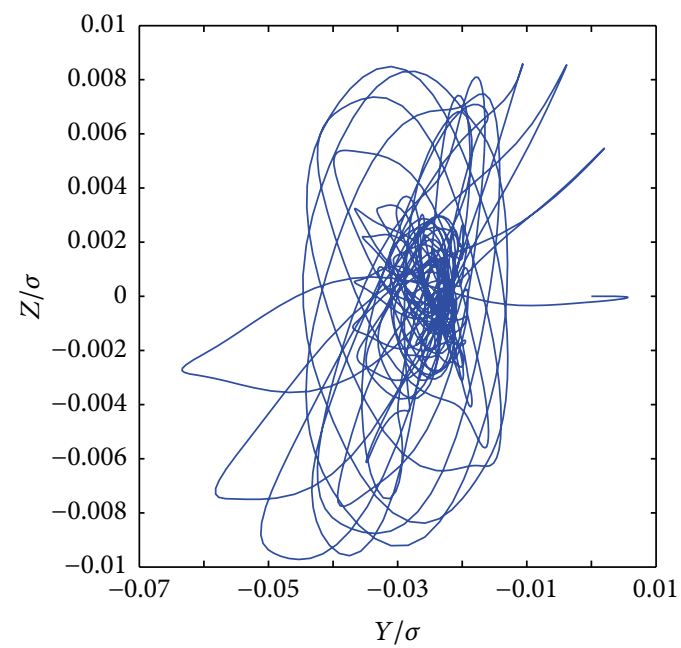

(1)

FIGURE 9: Orbit plots at different rotation speed ratio: (a) $p=0.6015$; (b) $p=0.6018$; (c); $p=0.6529$; (d) $p=0.6531$; (e) $p=1.3570$; (f) $p=1.3573$; (g) $p=1.4576$; (h) $p=1.4578$; (i) $p=0.7570$; (j) $p=0.7882$; (k) $p=0.7919$; (l) $p=0.8109$. 


\begin{tabular}{|c|c|}
\hline$o:$ & Center of disc \\
\hline$o-X Y Z:$ & Stationary coordinates \\
\hline$o \xi$ & Crack direction \\
\hline$o \xi \eta$ & $\begin{array}{l}\text { Rotating coordinates on the cross } \\
\text { section of cracked shaft }\end{array}$ \\
\hline$o X:$ & Axial direction of rotating shaft \\
\hline$o Y Z:$ & $\begin{array}{l}\text { Stationary cross section of cracked } \\
\text { shaft }\end{array}$ \\
\hline$\beta:$ & $\begin{array}{l}\text { Unbalanced oriented angle measured } \\
\text { from the crack direction }\end{array}$ \\
\hline$\Delta k_{\eta}:$ & $\begin{array}{l}\text { The reduce amount of the stiffness in } \\
\eta \text { direction }\end{array}$ \\
\hline$\Delta k_{\xi}:$ & $\begin{array}{l}\text { The reduce amount of the stiffness in } \\
\xi \text { direction }\end{array}$ \\
\hline$\lambda:$ & $\begin{array}{l}\text { Stability degree of the periodic } \\
\text { system }\end{array}$ \\
\hline$\mu_{i}:$ & Floquet multipliers \\
\hline$\Omega:$ & The rotation speed of rotor \\
\hline $\bar{Y}:$ & $\begin{array}{l}\text { Dimensionless rotor response in } \\
\text { vertical direction }\end{array}$ \\
\hline $\bar{Z}:$ & $\begin{array}{l}\text { Dimensionless rotor response in } \\
\text { horizontal direction }\end{array}$ \\
\hline$\sigma:$ & Static weight deflection \\
\hline$\varepsilon:$ & Mass eccentricity of disc \\
\hline$\Xi(\xi)=\operatorname{sign}(\xi):$ & Switching function for the crack \\
\hline$\zeta:$ & Dimensionless damping coefficient \\
\hline$a:$ & Crack depth \\
\hline$c:$ & Damping coefficient \\
\hline$C_{0}:$ & Flexibility of the uncracked shaft \\
\hline$C_{\eta}:$ & $\begin{array}{l}\text { Flexibility of the uncracked shaft in } \eta \\
\text { direction }\end{array}$ \\
\hline$C_{\xi}:$ & $\begin{array}{l}\text { Flexibility of the uncracked shaft in } \xi \\
\text { direction }\end{array}$ \\
\hline$D:$ & Diameter of the shaft \\
\hline$e=\varepsilon / \sigma$ & $\begin{array}{l}\text { Dimensionless mass eccentricity of } \\
\text { the disc }\end{array}$ \\
\hline$k_{0}:$ & The stiffness of the uncracked shaft \\
\hline$L:$ & Length of shaft \\
\hline$m:$ & Mass of disc \\
\hline$p:$ & Rotational speed ratio. \\
\hline
\end{tabular}

\section{Conflict of Interests}

The authors declare that they do not have any commercial or associative interest that represents a conflict of interests in connection with the work submitted.

\section{Acknowledgment}

This work is supported by National Natural Science Foundation of China (no. 11402087).

\section{References}

[1] R. Gasch, "A survey of the dynamic behaviour of a simple rotating shaft with a transverse crack," Journal of Sound and Vibration, vol. 160, no. 2, pp. 313-332, 1993.
[2] A. D. Dimarogonas, "Vibration of cracked structures: a state of the art review," Engineering Fracture Mechanics, vol. 55, no. 5, pp. 831-857, 1996.

[3] G. Meng and R. Gasch, "Stability and stability degree of a cracked flexible rotor supported on journal bearings," Journal of Vibration and Acoustics, Transactions of the ASME, vol. 122, no. 2, pp. 116-125, 2000.

[4] C. D. Untaroiu, A. Untaroiu, and M. Boiangiu, "Dynamic stability analysis of periodically time-varying rotor system with a transverse crack," Engineering, vol. 3, no. 7, pp. 719-725, 2011.

[5] C. Guo, M. A. AL-Shudeifat, J. Yan, L. A. Bergman, D. M. McFarland, and E. A. Butcher, "Stability analysis for transverse breathing cracks in rotor systems," European Journal of Mechanics A: Solids, vol. 42, pp. 27-34, 2013.

[6] Q. Han and F. Chu, "Parametric instability of a rotor-bearing system with two breathing transverse cracks," European Journal of Mechanics, A/Solids, vol. 36, pp. 180-190, 2012.

[7] Q. Han and F. Chu, "Parametric instability of a Jeffcott rotor with rotationally asymmetric inertia and transverse crack," Nonlinear Dynamics, vol. 73, no. 1-2, pp. 827-842, 2013.

[8] Q. K. Han, J. S. Zhao, and F. L. Chu, "Dynamic analysis of a geared rotor system considering a slant crack on the shaft," Journal of Sound and Vibration, vol. 331, no. 26, pp. 5803-5823, 2012.

[9] P. C. Muller, J. Bajkowski, and D. Soffker, "Chaotic motions and fault detection in a cracked rotor," Nonlinear Dynamics, vol. 5, no. 2, pp. 233-254, 1994.

[10] R. Genesio, M. Tartaglia, and A. Vicino, "On the estimation of asymptotic stability regions: state of the art and new proposals," IEEE Transactions on Automatic Control, vol. 30, no. 8, pp. 747755, 1985.

[11] S. C. Sinha and E. A. Butcher, "Solution and stability of a set of PTH order linear differential equations with periodic coefficients via Chebyshev polynomials," Mathematical Problems in Engineering, vol. 2, no. 2, pp. 165-190, 1996.

[12] N. Bachschmid and P. Pennacchi, "Crack effects in rotor dynamics," Mechanical Systems and Signal Processing, vol. 22, no. 4, pp. 761-762, 2008.

[13] N. Bachschmid, P. Pennacchi, and E. Tanzi, "A sensitivity analysis of vibrations in cracked turbo generator units versus crack position and depth," Mechanical Systems and Signal Processing, vol. 24, no. 3, pp. 844-859, 2010.

[14] N. Bachschmid, P. Pennacchi, and E. Tanzi, "Some remarks on breathing mechanism, on non-linear effects and on slant and helicoidal cracks," Mechanical Systems and Signal Processing, vol. 22, no. 4, pp. 879-904, 2008.

[15] N. Bachschmid and E. Tanzi, "Deflections and strains in cracked shafts due to rotating loads: a numerical and experimental analysis," International Journal of Rotating Machinery, vol. 10, no. 4, pp. 283-291, 2004.

[16] I. W. Mayes and W. G. R. Davies, "Analysis of the response of a multi-rotor-bearing system containing a transverse crack in a rotor," Journal of Vibration, Acoustics, Stress, and Reliability in Design, vol. 106, no. 1, pp. 139-145, 1984.

[17] Q. K. Han and F. L. Chu, "The effect of transverse crack upon parametric instability of a rotor-bearing system with an asymmetric disk," Communications on Nonlinear Science and Numerical Simulation, vol. 17, no. 12, pp. 5189-5200, 2012.

[18] D. J. Han, "Vibration analysis of periodically time-varying rotor system with transverse crack," Mechanical Systems and Signal Processing, vol. 21, no. 7, pp. 2857-2879, 2007. 
[19] T. H. Patel and A. K. Darpe, "Influence of crack breathing model on nonlinear dynamics of a cracked rotor," Journal of Sound and Vibration, vol. 311, no. 3-5, pp. 953-972, 2008.

[20] W. Lu and F. Chu, "Shaft crack identification based on vibration and AE signals," Shock and Vibration, vol. 18, no. 1-2, pp. 115-126, 2011.

[21] J.-J. Sinou and B. Faverjon, "The vibration signature of chordal cracks in a rotor system including uncertainties," Journal of Sound and Vibration, vol. 331, no. 1, pp. 138-154, 2012.

[22] Z. K. Peng, Z. Q. Lang, G. Meng, and F. L. Chu, "The effects of crack on the transmission matrix of rotor systems," Shock and Vibration, vol. 18, no. 1-2, pp. 91-103, 2011.

[23] B. Chomette, A. Fernandes, and J.-J. Sinou, "Cracks detection using active modal damping and piezoelectric components," Shock and Vibration, vol. 20, no. 4, pp. 619-631, 2013.

[24] R. T. Liong and C. Proppe, "Application of the cohesive zone model for the evaluation of stiffness losses in a rotor with a transverse breathing crack," Journal of Sound and Vibration, vol. 332, no. 8, pp. 2098-2110, 2013.

[25] S. C. Huang, Y. M. Huang, and S. M. Shieh, "Vibration and stability of a rotating shaft containing a transverse crack," Journal of Sound and Vibration, vol. 162, no. 3, pp. 387-401, 1993.

[26] L. Cheng, N. Li, X.-F. Chen, and Z.-J. He, "The influence of crack breathing and imbalance orientation angle on the characteristics of the critical speed of a cracked rotor," Journal of Sound and Vibration, vol. 330, no. 9, pp. 2031-2048, 2011.

[27] A. K. Darpe, K. Gupta, and A. Chawla, "Analysis of the response of a cracked Jeffcott rotor to axial excitation," Journal of Sound and Vibration, vol. 249, no. 3, pp. 429-445, 2002. 

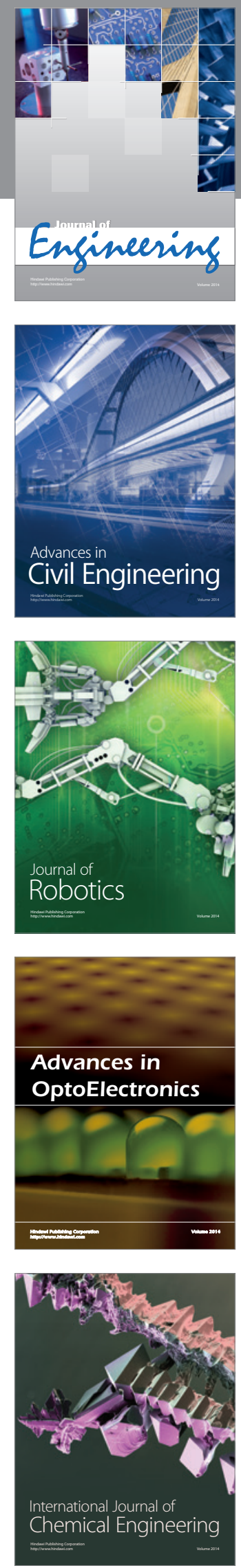

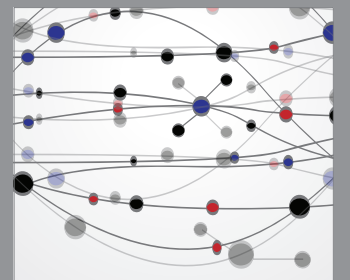

The Scientific World Journal
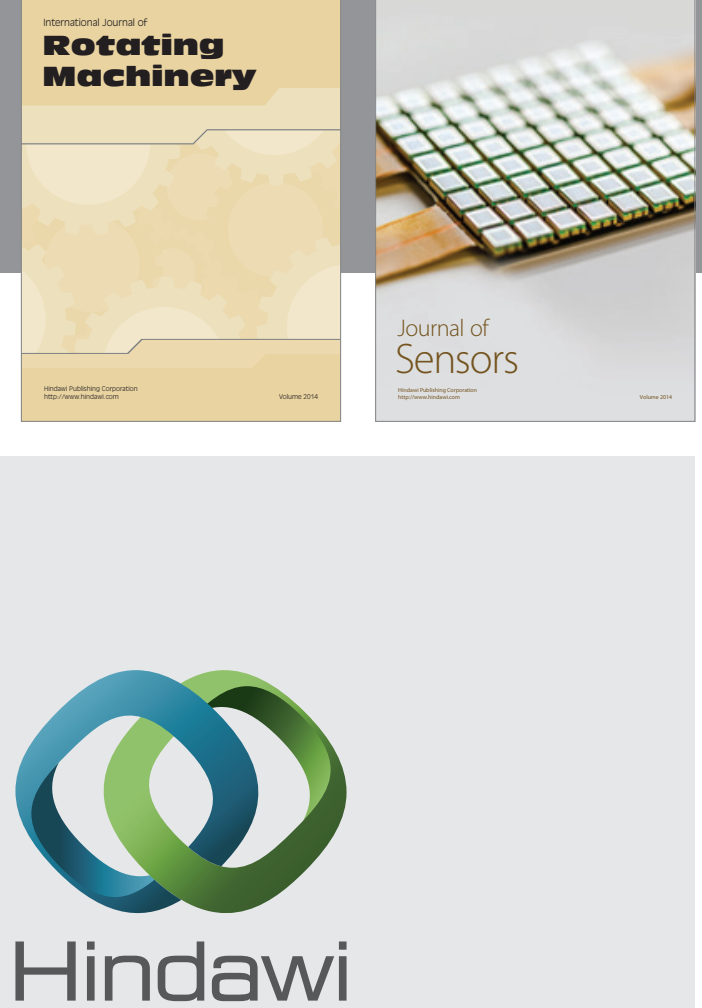

Submit your manuscripts at http://www.hindawi.com
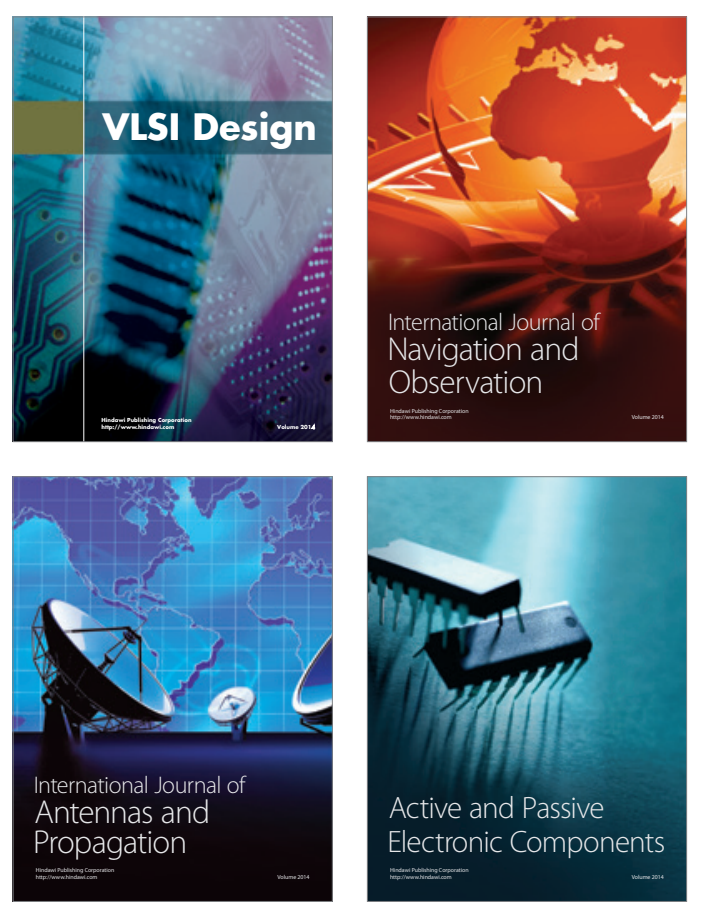
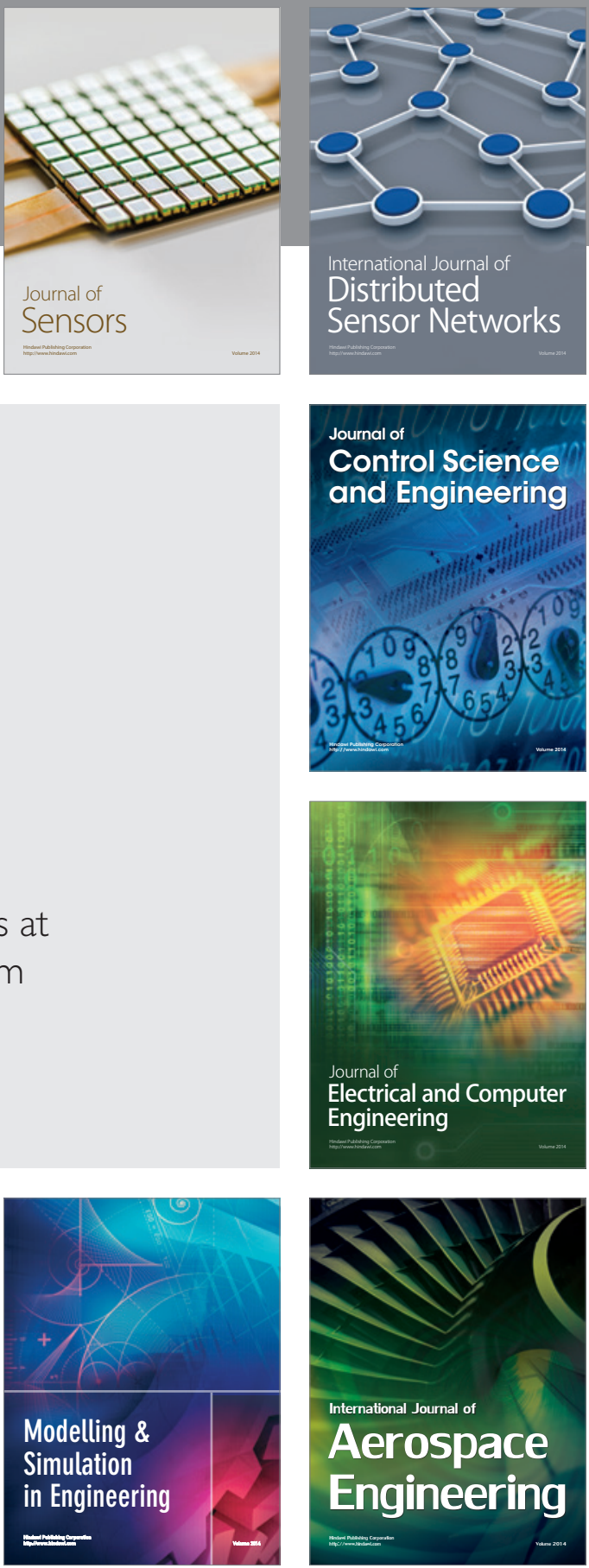

Journal of

Control Science

and Engineering
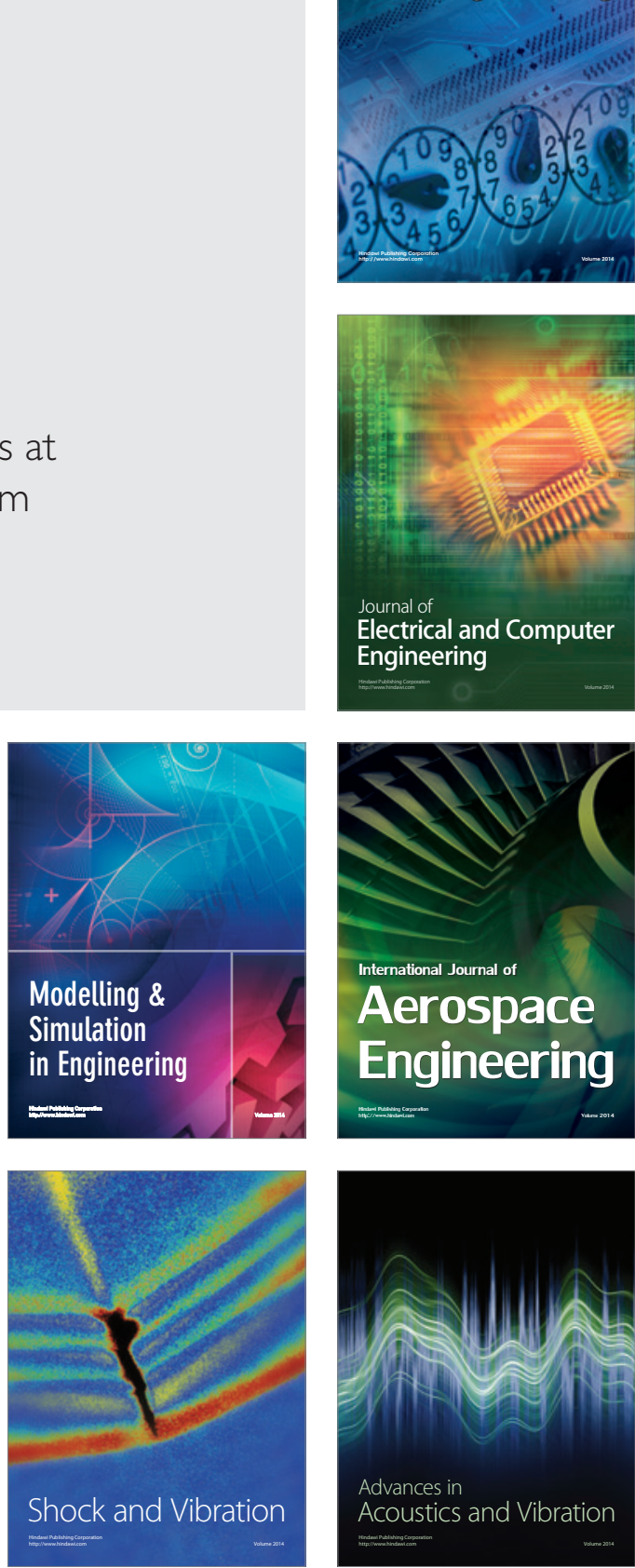